\title{
Integral field spectroscopy of the ultraluminous X-ray source Holmberg II X-1
}

\author{
I. Lehmann ${ }^{1}$, T. Becker ${ }^{2}$, S. Fabrika ${ }^{3}$, M. Roth ${ }^{2}$, T. Miyajii ${ }^{4}$, V. Afanasiev ${ }^{3}$, O. Sholukhova ${ }^{3}$, S. F. Sánchez ${ }^{2}$, \\ J. Greiner ${ }^{1}$, G. Hasinger ${ }^{1}$, E. Costantini ${ }^{1}$, A. Surkov ${ }^{3}$, and A. Burenkov ${ }^{3,5}$ \\ 1 Max-Planck-Institut für extraterrestrische Physik, Giessenbachstraße, PF 1312, 85741 Garching, Germany \\ e-mail: [ile;jcg;grh; elisa]@mpe.mpg.de \\ 2 Astrophysikalisches Institut Potsdam, An der Sternwarte 16, 14471 Potsdam, Germany \\ e-mail: [tbecker;mmroth; ssanchez]@aip.de \\ 3 Special Astrophysical Observatory of the Russian AS, Nizhnij Arkhyz 369167, Russia \\ e-mail: [fabrika;olga;vafan; sura;ban]@sao.ru \\ 4 Department of Physics, Carnegie Mellon University, Pittsburgh, PA 15213, USA \\ e-mail: miyaji@astro.phys.cmu.edu \\ 5 Isaac Newton Institute of Chile, SAO Branch, Russia
}

Received 9 December 2003 / Accepted 19 October 2004

\begin{abstract}
We present optical integral field observations of the H II region containing the ultraluminous X-ray source Holmberg II X-1. We confirm the existence of an X-ray ionized nebula as the counterpart of the source owing to the detection of an extended He II $\lambda 4686$ region $(21 \times 47 \mathrm{pc})$ at the Chandra ACIS-S position. An extended blue object with a size of $11 \times 14 \mathrm{pc}$ is coincident with the X-ray/He II $\lambda 4686$ region, which could indicate that it is either a young stellar complex or a cluster. We have derived an X-ray to optical luminosity ratio of $L_{X} / L_{B} \geq 170$, and presumable it is $L_{X} / L_{B} \sim 300-400$ using the recent HST ACS data. We find a complex velocity dispersion at the position of the ULX. In addition, there is a radial velocity variation in the X-ray ionized region found in the He II emission of $\pm 50 \mathrm{~km} \mathrm{~s}^{-1}$ on spatial scales of 2-3". We believe that the putative black hole not only ionizes the surrounding HII gas, but also perturbs it dynamically (via jets or the accretion disk wind). The spatial analysis of the public Chandra ACIS-S data reveals a point-like X-ray source and gives marginal indication of an extended component ( $\ll 15 \%$ of the total flux). The XMM-Newton EPIC-PN spectrum of HoII X-1 is best fitted with an absorbed power law in addition to either a thermal thick plasma or a thermal thin plasma or a multi-colour disk black body (MCD). In all cases, the thermal component shows a relatively low temperature $(k T \sim 0.14-0.22 \mathrm{keV})$. Finally we discuss the optical/X-ray properties of HoII X-1 with regards to the possible nature of the source. The existence of an X-ray ionized nebula coincident with the ULX and the soft X-ray component with a cool accretion disk favours the interpretation as an intermediate-mass black hole (IMBH). However, the complex velocity behaviour at the position of the ULX indicates a dynamical influence of the black hole on the local HII gas.
\end{abstract}

Key words. X-rays: ISM - ISM: HII regions - ISM: individual objects: Holmberg II X-1 - ISM: kinematics and dynamics instrumentation: spectrographs

\section{Introduction}

The two main classes of discrete galactic X-ray sources, X-ray binaries and supernova remnants (SNR), have been known and relatively well understood for decades. A third class of galactic X-ray sources was detected with Einstein thanks to its high spatial resolution and its large collecting area (Fabbiano 1989). These objects have become known as Ultraluminous X-ray sources (ULX) and they have 0.5-10 keV luminosities of $10^{39-41} \mathrm{erg} \mathrm{s}^{-1}$, generally higher than black hole binaries such as Cyg X-1 and LMC X-1, but lower than that of active galactic nuclei (AGN). Assuming Eddington luminosities, this corresponds to accretion onto black holes of between ten and several hundred solar masses suggesting intermediatemass black holes (IMBHs; see Colbert \& Mushotzky 1999; Miller \& Colbert 2004, for a review). ULX are not located in the dynamical center of their host galaxies and thus they are not caused by sub-Eddington accretion onto a central AGN-type super-massive black hole. The identification of the optical counterparts of ULX is essential to determine the nature of these objects. The number of optically identified ULX is still limited, only a small number of reliable optical counterparts are known to date. Several ULX seem to be associated with H II regions or nebula (e.g., Pakull \& Mironi 2002; Foschini et al. 2002; Wang 2002). However, some ULX have been 
Table 1. Instruments and observations.

\begin{tabular}{|c|c|c|c|c|}
\hline \multirow[b]{2}{*}{$\begin{array}{l}\text { Telescope } \\
\text { instrument }\end{array}$} & \multicolumn{2}{|c|}{ integral field spectroscopy } & \multirow{2}{*}{$\begin{array}{l}\text { long-slit spectroscopy } \\
\text { SAO } 6 \mathrm{~m} \\
\text { LSS }\end{array}$} & \multirow{2}{*}{$\begin{array}{l}\text { imaging } \\
\text { CFHT } 3.6 \mathrm{~m} \\
\text { OSIS } \\
\end{array}$} \\
\hline & $\begin{array}{l}\text { Calar Alto } 3.5 \mathrm{~m} \\
\text { PMAS }\end{array}$ & $\begin{array}{l}\text { SAO } 6 \mathrm{~m} \\
\text { MPFS }\end{array}$ & & \\
\hline Date of observation & 28.10 .2001 & 15.03 .2002 & $14-15.01 .2002$ & 11.03 .2000 \\
\hline Exposure time & $4 \times 900$ s mosaic, - offsets: $4^{\prime \prime}$ & $2 \times 900 \mathrm{~s}$ & $\begin{array}{l}1800 \mathrm{~s}(\mathrm{~N} 1-3) \\
2700 \mathrm{~s}(\mathrm{~N} 4-5)\end{array}$ & $\begin{array}{l}2 \times 1200 \mathrm{~s}(\mathrm{H} \alpha) \\
2 \times 300 \mathrm{~s}(R) \\
2 \times 500 \mathrm{~s}(B)\end{array}$ \\
\hline Seeing & $1.0^{\prime \prime}$ & $1.5-2.0^{\prime \prime}$ & $1.5^{\prime \prime} / 1.1^{\prime \prime}$ & $\sim 0.7^{\prime \prime}$ \\
\hline Field of view & $8^{\prime \prime} \times 8^{\prime \prime}, 12^{\prime \prime} \times 12^{\prime \prime}($ mosaic $)$ & $16^{\prime \prime} \times 16^{\prime \prime}$ & $2^{\prime \prime} \times 140^{\prime \prime}$ & $3^{\prime} \times 3^{\prime}$ \\
\hline Pixel scale & $0.5^{\prime \prime} / \mathrm{pix}$ & $1.0^{\prime \prime} / \mathrm{pix}$ & $0.41^{\prime \prime} /$ pix & $0.088^{\prime \prime} / \mathrm{pix}$ \\
\hline Image size in pixels & $2048 \times 4096($ binned $2 \times 2)$ & $1024 \times 1024$ & $1024 \times 1024$ & $2048 \times 2048$ \\
\hline Spectral coverage & $4450-5140 \AA$ & $4210-6820 \AA$ & $4330-6750 \AA$ & - \\
\hline Dispersion & $0.76 \AA$ A/binned pix & $2.7 \AA /$ pix & $2.4 \AA /$ pix & - \\
\hline Spectral resolution & $1.5 \AA$ & $7.0 \AA$ & $7.2 \AA$ & - \\
\hline
\end{tabular}

associated with accreting black holes in globular clusters (see Angelini et al. 2001; Wu et al. 2002). The optical counterpart to an extremely luminous X-ray source near Holmberg IX is a shock-heated nebulae, which is associated with an optically faint non-stellar source (Miller 1995). The discovery of the intense He II $\lambda 4686$ nebular recombination line in Holmberg II X-1 (hereafter; HoII X-1) indicates that the interstellar medium probably reprocesses part of the X-ray luminosity of $\sim 10^{40} \mathrm{erg} \mathrm{s}^{-1}$. Assuming quasi-isotropic emission (Pakull \& Mironi 2002) and a distance $D=3.2 \mathrm{Mpc}$, the X-ray luminosity of HoII X-1 corresponds to an Eddington mass of $\approx 80 M_{\odot}$, which is considered a rather strict lower limit to the mass of the accreting compact object if the X-ray emission is isotropic.

A more accurate distance to HoII of $3.39 \mathrm{Mpc}$ was recently determined by Karachentsev et al. (2002). The new distance estimate would result in only a minimal difference in the size, flux, and luminosity values we have calculated assuming a value of $D=3.2 \mathrm{Mpc}$.

The ROSAT HRI and PSPC data of HoII X-1 have been presented in detail by Zezas et al. (1999), revealing a pointlike, variable source (on scales of days and years) at the edge of the compact H II region \#70 (Hodge et al. 1994). The ROSAT PSPC spectrum was best described by either a steep power law with $\Gamma=2.7$ or a thermal plasma with $k T \sim 0.8 \mathrm{keV}$. Miyaji et al. (2001; hereafter MLH01) found that the ASCA spectrum extends to harder energies. The hard part of the spectrum is best fitted with a flatter power law with $\Gamma \sim 1.9$ and intrinsic absorption above that of our galaxy. A multi-colour disk blackbody model (MCD, Mitsuda et al. 1984) did not fit the ASCA spectrum of HoII X-1, unlike some other ULX of similar luminosities. A joint PSPC-ASCA spectral analysis showed a soft excess component above the power law component. The soft excess could be described by either an MCD model with $k T_{\text {in }}=0.17 \mathrm{keV}$, or a thin thermal plasma with $k T=0.3 \mathrm{keV}$. MLH01 disfavored the MCD interpretation for the soft excess based on the large discrepancy between the black hole mass estimated from $k T_{\text {in }}$ and that estimated from the normalization. Furthermore, the spatial analysis of the ROSAT HRI image indicates an extended component.
In this paper we report on optical integral field and long-slit spectroscopic observations of the ultraluminous X-ray source $\mathrm{X}-1$ in Holmberg II, as well as on results from the spatial and spectral analysis of the public XMM-Newton and Chandra data.

The outline of the paper is as follows. The optical imaging, and the long-slit and integral field spectroscopy of HoII X-1 are presented in Sect. 2. The H II region associated with the Xray source is presented in Sect. 3. The optical properties (e.g., emission line flux maps, velocity dispersion, and radial velocities) of the H II region are described in Sect. 4. In Sect. 5 we present the X-ray spectral and spatial analysis based on public XMM-Newton EPIC-PN and Chandra ACIS-S data. The implications of our results for the nature of the ULX HoII X-1 are discussed in Sect. 6.

\section{Observations and data reduction}

We have carried out integral field observations with the Potsdam Multi-Aperture Spectrophotometer (PMAS; Roth et al. 2000) and with the Multi-Pupil Fiber Spectrograph (MPFS; Afanasiev et al. 1995a), and long-slit observation with the Long-Slit Spectrograph (LSS; Afanasiev et al. 1995b) to determine the nature of the optical counterpart of HoII X-1. These observations are complementary because of the different technical characteristics of each instrument, e.g.; FOV, pixel scale, and spectral resolution, and because of the observational conditions (see Table 1). Because the accurate Chandra position (see Sect. 5) was not known at the time of our observations, it was especially necessary to cover a large field of view with the integral field technique. Figure 1 shows the overlays of the FOV for each instrument on the CHFT archival $\mathrm{H} \alpha$ image (see Sect. 2.3) of the HoII X-1 region. A detailed description of the observation is given below.

\subsection{Integral field spectroscopy with PMAS and MPFS}

We have used the PMAS at the Calar Alto $3.5 \mathrm{~m}$ Telescope to obtain integral field observations of the optical counterpart of HoII X-1. The observations were part of a Science Verification run from October 23-28, 2001. On Oct. 28 we observed a set of 


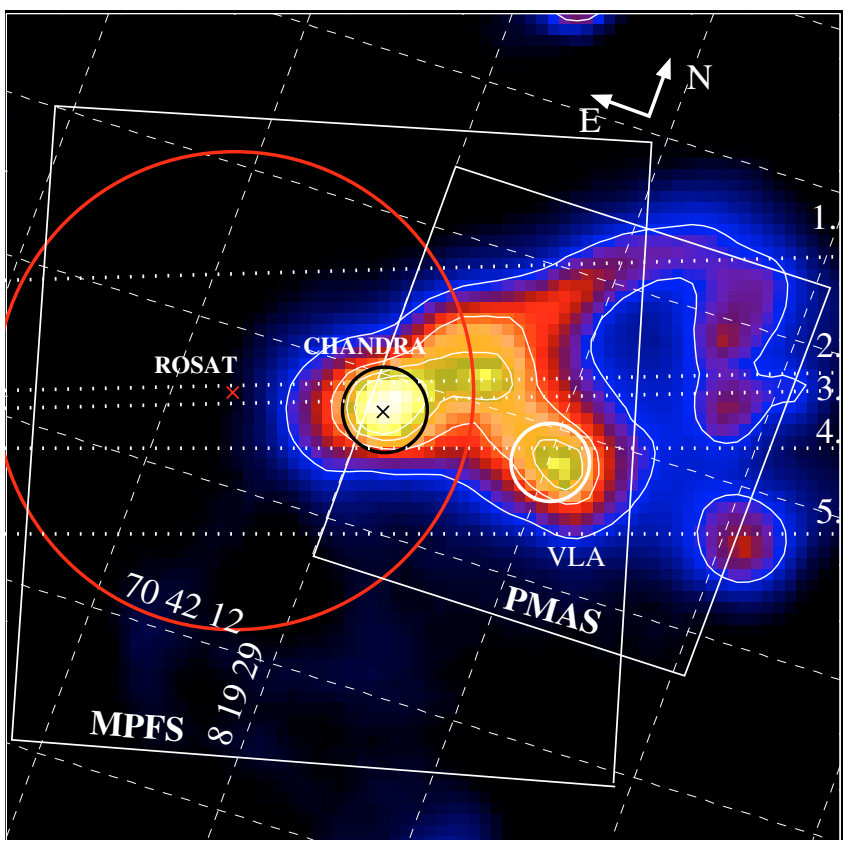

Fig. 1. CFHT archival $\mathrm{H}_{\alpha}$ image of the HoII X-1 region. The MPFS FOV and the PMAS mosaic FOV are superposed. The small black and the large red circles give the $90 \%$ confidence circles of the Chandra ACIS-S (RA: $8^{\mathrm{h}} 19^{\mathrm{m}} 29.0^{\mathrm{s}}$, Dec: $+70^{\circ} 42^{\prime} 19.0^{\prime \prime} ; \mathrm{J} 2000$ ) and the ROSAT HRI positions, respectively. The small white circle shows the location of the radio peak at 6,20 , and $90 \mathrm{~cm}$ wavelengths (see Tongue \& Westpfahl 1995). Long-slit positions (1-5) are indicated with dotted lines. The overlaid coordinate grid is given in steps of $1^{\mathrm{s}}$ in RA and in steps of 4 " in Dec.

4 mosaic pointings, each offset by $4 \times 4$ arsec in four different directions from the previous deep field in order to search for spectral signatures which could be associated with HoII X-1.

Motivated by the detection of the He II emission at the edge of the PMAS mosaic FOV (see Sect. 4.1) we conducted further observations with MPFS at the 6-m SAO telescope in Russia. The LSS observations, obtained before the MPFS data (see Table 1), provided valuable insight so as point the MPFS instrument exactly on the He II region. The "heel" of the foot-like H II region HSK \#70 (Hodge et al. 1994) was centered on the CCD frame during the MPFS observations (Fig. 1).

The PMAS and MPFS data were reduced using P3d, an IDL-based data reduction package developed at the Astrophysikalisches Institut Potsdam (Becker 2002). The bias was subtracted using a bias exposure taken at the beginning of each night. Continuum lamp and mercury (PMAS) or neon (MPFS) emission line lamp exposures were taken before and after the science exposures. The continuum lamp exposures were used to trace the individual spectra.

The line lamp exposures were used for wavelength calibration. The small number of mercury lines in the PMAS calibration spectra does not allow a very accurate wavelength calibration. Therefore we have not derived radial velocities from the PMAS data.

The fiber throughput variations were calibrated using a sky flat taken at the end of the night. The absolute flux calibration of the PMAS and MPFS spectra was obtained using standard star exposures of HR153 and G191B2B, respectively, just before the science exposures.

The PMAS images were combined into a single mosaic frame after correcting for atmospheric refraction (Filippenko 1982), in each individual exposure.

\subsection{Long-slit observations}

Observations were carried out with the LSS instrument installed at the prime focus of the 6-m SAO telescope. The slit orientation for the LSS spectra (N1-5) was along the main axis of the foot-like $\mathrm{H}$ II region, $\mathrm{PA} \approx 120^{\circ}$ (see Fig. 1 ). The relative offsets between slits $\mathrm{N} 2$ and N3, and between slits N3 and N4, are $0.6^{\prime \prime}$ and 1.3", respectively. Slit N1 was offset 3.3" NorthEast of N2, and slit N5 was offset 2.5" South-West of N4.

The accurate locations of the slits were determined using images from the TV-guiding camera on the LSS spectrograph. The FOV of the guiding camera is $2^{\prime}$. Five stars surrounding the target were used to determine the coordinates of the slit in each image.

We used standard MIDAS procedures to reduce the LSS spectra. The wavelength calibration was checked again using the $[\mathrm{O} \mathrm{I}] \lambda 5577$ and $\lambda 6300$ sky lines.

\subsection{CFHT BRH $\alpha$ archival imaging data}

We adopted the accurate Chandra coordinates and applied linear astrometry to the CFHT $\mathrm{H} \alpha, R$, and $B$ archive images to determine the position of the X-ray source in the H II region.

For an absolute photometric calibration two images of the globular cluster NGC 4147 taken during the same night were used. Six standard stars from the cluster (Odewahn et al. 1992) were used to calibrate the images. The calibration was double checked by independent measurements of the comparison stars in $B$-band CCD images made by V. Goranskii with the 1-m SAO telescope.

\section{Optical counterpart of the ULX Holl X-1}

To obtain reliable astrometry of the CFHT $\mathrm{H} \alpha, R$, and $B$ archival images we used the USNO-B1.0 catalogue. The astrometric accuracy of the catalogue is about $0.2^{\prime \prime}$ at J2000. About fifteen USNO-B1.0 stars were found within the OSIS FOV around HoII X-1. The standard deviations of the star positions are $0.20^{\prime \prime}$ for the $\mathrm{H} \alpha, 0.13^{\prime \prime}$ for the $B$, and $0.15^{\prime \prime}$ for the $R$ images. In Fig. 2 we present the CFHT images with the Chandra ACIS-S source position marked (see detailed description in Sect. 5). An object on the $B$ image coincides perfectly with the position of the X-ray source. Its magnitude is $B=20.5 \pm 0.1 \mathrm{mag}$.

Since all the stars on the $B$ image in Fig. 2 look elongated, while the seeing was fairly good $\left(\approx 0.7^{\prime \prime}\right)$, we fitted 2D-Gaussians to 23 stars surrounding the X-ray position. The elongation of these stars was $b / a=1.36 \pm 0.05$, where the major axis size is $b=0.89 \pm 0.04$ " and the minor axis is $a=0.65 \pm 0.01^{\prime \prime}$. The position angle of the star images was $\mathrm{PA}=-19.2 \pm 3.0^{\circ}$. 


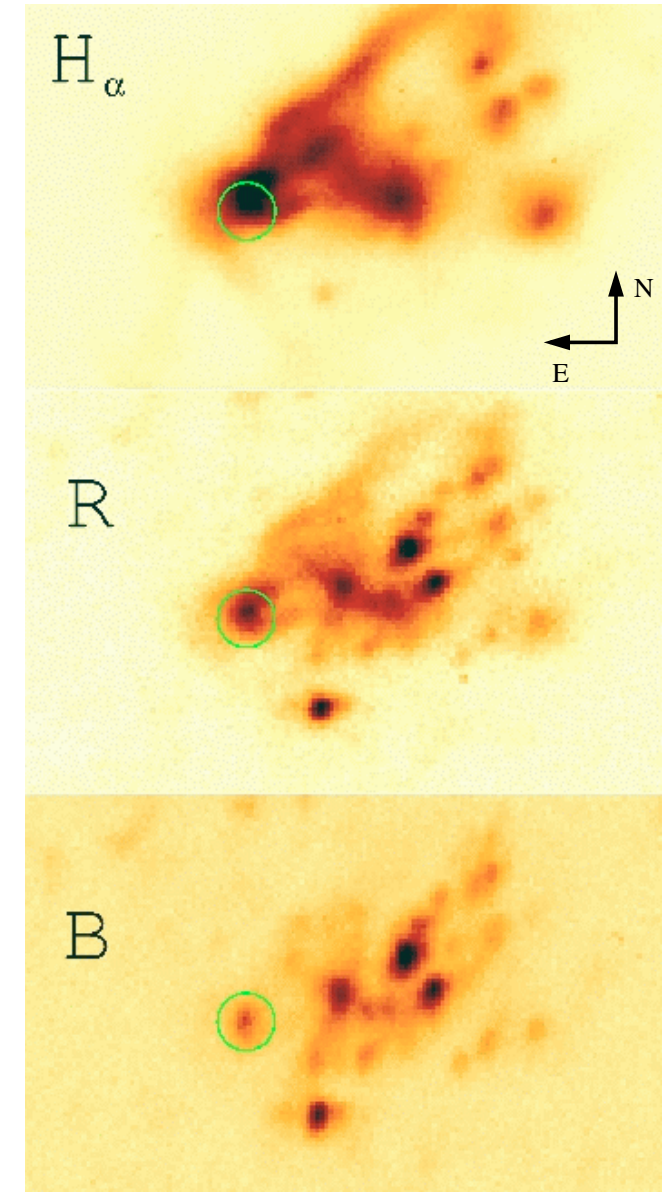

Fig. 2. CFHT $\mathrm{H} \alpha, R$, and $B$ images of the HoII $\mathrm{X}-1$ region with the marked Chandra position (a circle $1^{\prime \prime}$ in radius) of the X-ray source. The image size is $24.5^{\prime \prime} \times 14.0^{\prime \prime}$.

The object coincident with the X-ray source has the following parameters: $b / a \approx 1.29, b \approx 1.22^{\prime \prime}, a \approx 0.96^{\prime \prime}$ and $\mathrm{PA} \approx-10^{\circ}$, which indicates that in comparison with surrounding stars the counterpart is an extended object, e.g. a nebula or a compact stellar cluster. Its intrinsic size is about $0.69^{\prime \prime}$ $(11 \mathrm{pc})$ in the West-East direction and about $0.85^{\prime \prime}(13 \mathrm{pc})$ in the North-South direction. The orientation is PA $\sim 0^{\circ}$.

Assuming the $B$-band flux calibrations from Allen (1973), and a line-of-sight extinction to HoII of $A_{B}=0.14^{\mathrm{m}}$ (Schlegel et al. 1998), the optical luminosity of the counterpart is estimated to be $L_{B} \sim 6 \times 10^{37} \mathrm{erg} / \mathrm{s}$. The absolute magnitude of this object is $M_{B}=-7.2$. Using the $\mathrm{X}$-ray luminosity in the 0.3-8.0 keV energy band of $L_{\mathrm{x}} \sim 10^{40} \mathrm{erg} / \mathrm{s}$, corrected for absorption (see Sect. 5.2), we find $L_{\mathrm{x}} / L_{B} \geq 170$. Recent $H S T$ ACS observations of HoII X-1, (published after the submission of this paper), resolved this object into several young stars (Kaaret et al. 2004), which agrees with our interpretation. Owing to the superb angular resolution of the ACS images Kaaret found a bright, point-like optical counterpart consistent either with a star with spectral type between $\mathrm{O} 4 \mathrm{~V}$ and $\mathrm{B} 3 \mathrm{Ib}$, or reprocessed emission from an X-ray illuminated accretion disk. Because the star found by Kaaret et al. (2004) is about one magnitude fainter than our extended blue counterpart this results in a $L_{\mathrm{x}} / L_{B}$ of $\sim 300-400$.

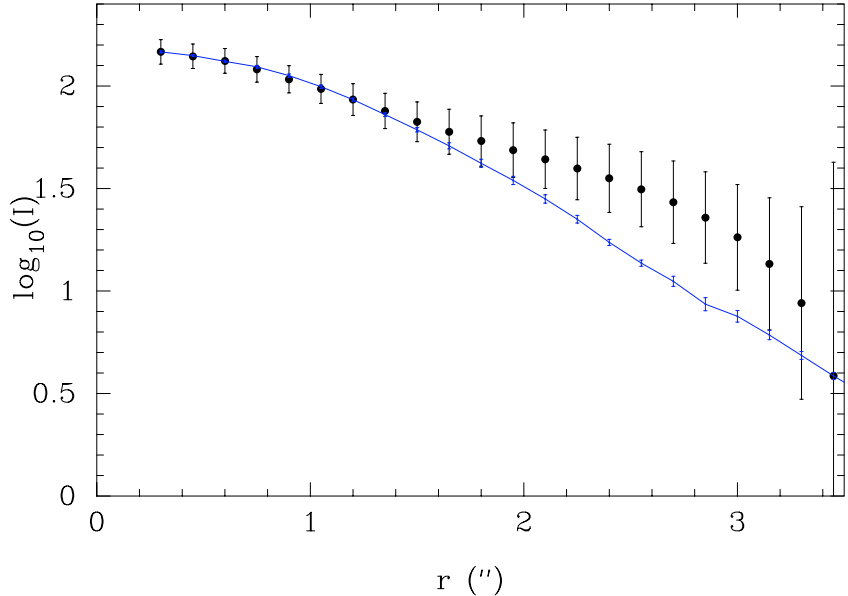

Fig. 3. Surface brightness profile of the He II emission around the ULX (solid circles) together with the MPFS PSF (solid line). The error bars include both the photon noise and the error in the surface brightness determination. The He II region around the ULX is clearly extended to about $3^{\prime \prime}$.

The MPFS He II $\lambda 4686$ line flux map (see Fig. 6 in Sect. 4.1) clearly shows a He II emission line region at the Chandra ACIS-S position, and coincident with the optical counterpart detected on the CHFT $B$ image. This confirms the classification of ULX HoII X-1 as an X-ray ionized nebula (Pakull \& Mirioni 2002). In order to determine if the He II emission is extended we derived the surface brightness profile of the He II region, using an algorithm based on Jedrzejewski (1987). This method increases the signal-to-noise in the outer part of the surface brightness profile, since it takes an average of the brightness along the eccentric anomaly. A similar technique is extensively used for the detection of host galaxies in QSOs (e.g., Sánchez \& Gonzalez-Serrano 2003).

The MPFS PSF was built using the continuum emission at a wavelength range near the He II emission. Figure 3 shows the surface brightness profile of the He II region together with the surface brightness profile of the PSF, scaled to the peak of the $\mathrm{He}$ II emission. The He II emission is clearly extended beyond $r \sim 2^{\prime \prime}$, which is also confirmed by the HST ACS He II narrow band image of Kaaret et al. (2004).

We find an extended and elongated He II $\lambda 4686$ region with nearly the same positional angle of PA $\sim-19^{\circ}$ as found for the blue counterpart on the $B$ image. The size of the He II $\lambda 4686$ region after PSF correction is about $1.4 \times 3.0^{\prime \prime}$ (see Fig. 3), which corresponds to about $21 \times 47 \mathrm{pc}$. The larger size of the He II region compared with the blue counterpart $(11 \times 14 \mathrm{pc})$ could be considered an argument for a stellar complex, where the $B$ band counterpart represents the continuum emission and the He II region represents the high excitation nebula.

The most important observational results from this section are that the He II emission is extended, in the same positional angle as the extended blue counterpart, and centered on the $\mathrm{X}$-ray source. This confirms the classification of HoII X-1 as an X-ray ionized nebula as suggested by Pakull \& Mirioni (2002). 


\section{Emission line properties}

To determine the physical parameters (e.g. the radial velocities and the velocity dispersions) of the $\mathrm{H}$ II region associated with the ULX, we have measured the properties of all emission lines in the LSS, PMAS and MPFS spectra. Whereas the PMAS spectra cover only the He II $\lambda 4686, \mathrm{H} \beta \lambda 4861$ and the [O III] $\lambda \lambda 4959,5007$ emission lines, the MPFS spectral range includes the $\mathrm{H} \gamma \lambda 4363$ and $\mathrm{H} \alpha \lambda 6563$ emission line regions as well.

Each emission line in the 240 MPFS and 506 PMAS integral field spectra was fitted with a Gaussian profile, applying the Levenberg-Marquardt algorithm (Press et al. 1992). The four adjustable parameters were the total line flux, the mean wavelength, the sigma width of the Gaussian, and the flux of the local linear continuum. The Levenberg-Marquardt algorithm also estimates one-sigma errors for each parameter. We considered only the emission line parameters of those lines which were at least $5 \sigma$ detections in the PMAS spectra, or $3 \sigma$ in the MPFS spectra. We visually checked the reliability of the chosen line detection thresholds for each spectrum.

\subsection{Line fluxes and He II line luminosity}

The emission line flux maps of $\mathrm{H} \beta$ and [O III] $\lambda \lambda 4959,5007$ derived from the PMAS mosaic spectra, and of $\mathrm{He}$ II, $\mathrm{H} \beta$, [O III] $\lambda \lambda 4959,5007, \mathrm{H} \alpha$ and [S II] $\lambda 6716$ derived from the MPFS spectra, are presented in the upper panel of Figs. 4, 6 and 7. The linear flux scale given below the maps starts with the blue color. The black regions mark the non-detection of a $5 \sigma$ line for PMAS spectra and of a 3 sigma line for MPFS spectra. The circle with $1^{\prime \prime}$ radius marks the X-ray position determined from the Chandra ACIS-S data (see Sect. 5).

Because the PMAS data were taken under better seeing conditions and because of the better angular sampling of PMAS (see Table 1), the PMAS emission line flux maps of $\mathrm{H} \beta$ and [O III] $\lambda 5007$ show more details than the MPFS maps. For instance, the peaks of both lines in the MPFS data are clearly resolved into two clumps with PMAS.

He II emission lines are clearly detected from a relatively compact region inside the $\mathrm{H}$ II region $\# 70$ at the Chandra ACIS-S position (see Fig. 6). He II emission lines are not detected in the individual PMAS spectra because of the short exposure time. However, the He II line is clearly seen in the co-added PMAS spectrum (see Fig. 5). In addition, the coadded spectrum is suggestive of an increasing blue continuum at wavelengths below $4700 \AA$, which is probably produced by the young stars resolved with HST. The increasing continuum to the blue is also seen in the LSS spectrum (see Fig. 8). The absolute continuum fluxes of the PMAS and LSS spectra are not comparable because of the different amount of sky background emission. Nevertheless, the increasing blue continuum cannot be explained by the sky background.

The fluxes of all but the He II $\lambda 4686$ emission lines peak outside the X-ray error circle (see Figs. 6 and 7). The peak PMAS fluxes agree well with the peak MPFS fluxes.

In Fig. 8 we present an averaged LSS spectrum of N2 and N3 at the location of the blue extended counterpart. This
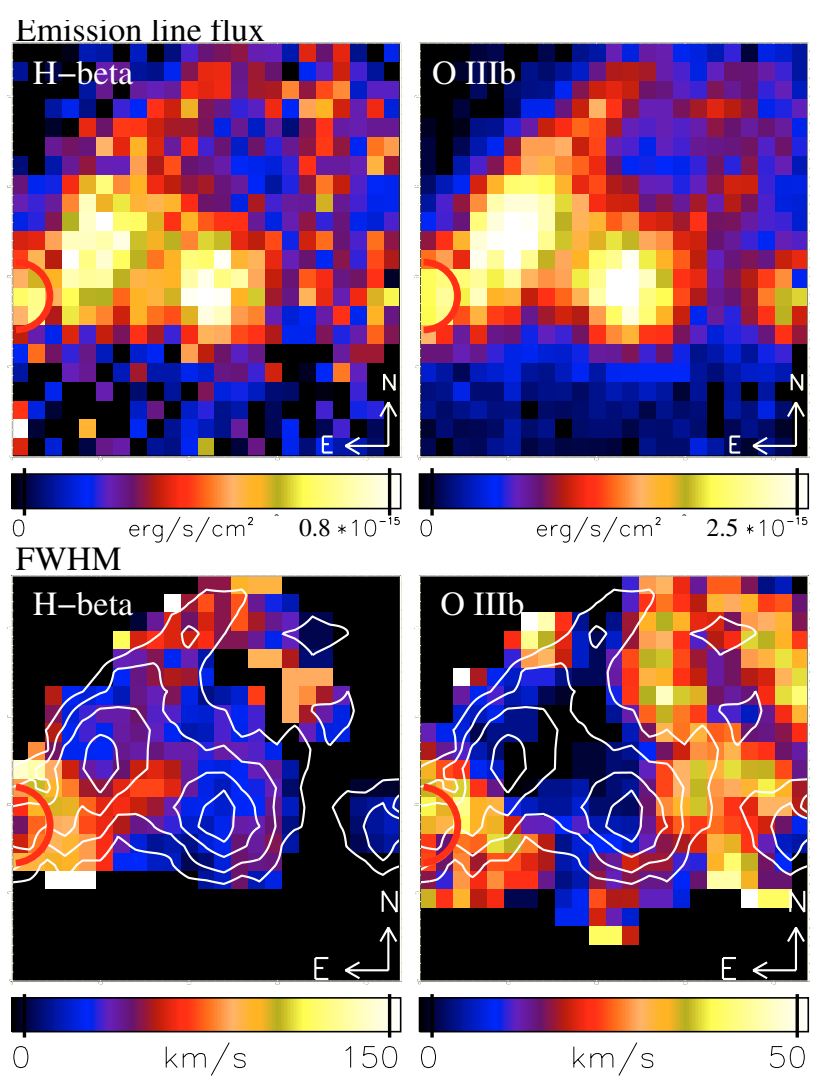

Fig. 4. PMAS emission line flux maps (upper panels), and emission line FWHM maps (lower panels), corrected for instrumental resolution, for $\mathrm{H} \beta$ and for [O III] $\lambda 5007$ in the HolI X-1 region. The $F W H M$ images have been smoothed using a $3 \times 3$ pixel box. The emission line flux contour of [O III] $\lambda 5007$ is overlaid on the FHWM maps. The ACIS-S error circle of the X-ray source position is shown. The FOV of the single images is about $11^{\prime \prime} \times 11.5^{\prime \prime}$. The black color in the flux maps and in the FWHM maps mark regions with no significant line detections and unresolved lines, respectively. The white color in the same maps indicates values above the limit given in the color bars.

spectrum covers the brightest region in He II; however, the spectrum did not cover the region completely. The real flux could be a factor of $\sim 1.5$ times larger. The brightest lines in the spectrum are the hydrogen lines and [O III] $\lambda \lambda 4959,5007$. No noticeable absorption lines or broad wings in the permitted lines are observed; however, the faint blue continuum is clearly seen. All significantly detected lines are narrow, and formed in the nebula. He II $\lambda 4686$ emission is the strongest permitted line (after the hydrogen lines) indicating a high excitation of the X-ray ionized nebula (XIN, Pakull \& Mirioni 2002).

Relative intensities of emission lines in the LSS spectra agree very well with those from the MPFS/PMAS spectra; however, they are slightly different in individual LSSspectra. The latter implies that the physical conditions of the gas may be different in different parts of the XIN. The relative fluxes of the MPFS spectra and the averaged LSS spectra N2 and $\mathrm{N} 3$ in units of the $\mathrm{H} \beta$ flux are given in Table 2 .

From the He II $\lambda 4686$ flux map in Fig. 6 we have determined a total line flux of $1.55 \times 10^{-15} \mathrm{erg} \mathrm{s}^{-1} \mathrm{~cm}^{-2}$ corresponding to a luminosity of $\sim 1.9 \times 10^{36} \mathrm{erg} \mathrm{s}^{-1}$. Using the averaged LSS spectra N2 and N3 at the X-ray source position we 


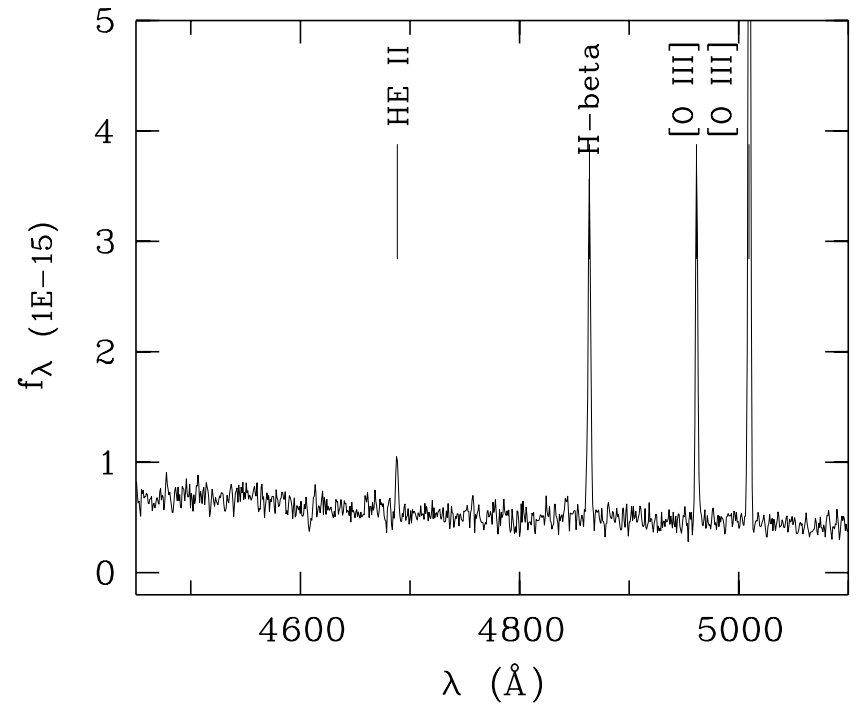

Fig. 5. The co-added PMAS spectrum at the X-ray position (see Fig. 4) clearly shows an He II $\lambda 4686$ emission line.

have determined a total He II $\lambda 4686$ luminosity of $L(\mathrm{He}$ II $) \approx$ $1.4 \times 10^{36} \mathrm{erg} \mathrm{s}^{-1}$, where the He II equivalent width is $29.7 \AA$. Furthermore, we have estimated the total He II $\lambda 4686$ flux from the monochromatic MPFS images in Fig. 10. Assuming a $5^{\prime \prime} \times 5^{\prime \prime}$ area of the He II region (not PSF corrected) we can directly measure the mean flux of $\log f_{\mathrm{He} \text { II }} \sim-16.1$, which gives a luminosity of $L \approx 2.2 \times 10^{36} \mathrm{erg} \mathrm{s}^{-1}$. The total $\mathrm{H} \beta$ luminosity in our LSS data is $L(\mathrm{H} \beta) \approx 9.5 \times 10^{36} \mathrm{erg} / \mathrm{s}$.

The He II luminosity agrees with the results published by Pakull \& Mirioni (2002) of $L(\mathrm{He}$ II $) \approx 2.5 \times 10^{36} \mathrm{erg} / \mathrm{s}$, and by Kaaret et al. (2004) of $L(\mathrm{He}$ II $) \approx 2.7 \times 10^{36} \mathrm{erg} / \mathrm{s}$ (keeping in mind that our long-slits do not cover the entire He II region).

\subsection{Diagnostic emission line flux ratios}

In order to understand the nature of the ionization inside the $\mathrm{H}$ II region \#70 we determined the emission line flux ratios from the LSS and MPFS data. Table 3 gives the diagnostic ratios derived from the averaged LSS at positions N2 and N3 (see Fig. 8). The MPFS line ratio maps of [O III] $\lambda 5007 / \mathrm{H} \beta$, [S II] $\lambda 6717 / \mathrm{H} \alpha$, and $\mathrm{H} \alpha / \mathrm{H} \beta$ are shown in Fig. 9.

The diagnostic line flux ratios ([O III] $\lambda 5007 / \mathrm{H} \beta \lambda 4861$, $\left[\begin{array}{ll}\mathrm{O} & \mathrm{I}\end{array}\right] \lambda 6300 / \mathrm{H} \alpha \quad \lambda 6563,[\mathrm{~N}$ II] $\lambda 6583 / \mathrm{H} \alpha \quad \lambda 6563$, and [S II] $\lambda 6716+\lambda 6731 / \mathrm{H} \alpha \lambda 6563$ see Table 3 ) derived from the averaged LSS spectrum and the MPFS line ratio maps agree well with the H II region classifications (Veilleux \& Osterbrock 1987). The [N II] $\lambda 6583 / \mathrm{H} \alpha \lambda 6563$ ratio is at the lower end for H II regions (see Fig. 12.1 in Osterbrock 1989).

The line flux ratio of [S II] $\lambda 6716+\lambda 6731 / \mathrm{H} \alpha$ is well below 0.4 (see Table 3), which is an indication that the emission comes from ionized gas in $\mathrm{H}$ II regions or in a nebula rather than from a supernova remnant (Smith et al. 1993). The 6, 20, and $90 \mathrm{~cm}$ radio data from Tongue \& Westpfahl (1995) show a peak at the position: $8^{\mathrm{h}} 19^{\mathrm{m}} 28^{2},+70^{\mathrm{deg}} 42^{\prime} 19^{\prime \prime}(\mathrm{J} 2000)$, which is about $3^{\prime \prime}$ West of the Chandra position (see Fig. 1). However, because of the low angular resolution of the data $\left(\sim 15^{\prime \prime}\right)$ the radio position is not precise enough to exclude a coincidence with the X-ray source. While the steep radio spectral index of $\alpha_{L C}=-0.5 \pm-0.1$ (between the 6 and $20 \mathrm{~cm}$ wavelength bands) favours a supernova remnant (SNR) as the source of the radio emission, the optical emission line ratios suggest that the region of the radio emission is instead a $\mathrm{H}$ II region.

The MPFS line ratio maps of [O III] $\lambda 5007 / \mathrm{H} \beta$ and [S II] $\lambda 6717 / \mathrm{H} \alpha$ in Fig. 9 do not show a large variation over the H II region. Inside the Chandra ACIS-S error circle and inside a region $\sim 10^{\prime \prime}$ North-West of that position the MPFS flux ratio map shows slightly larger values of the [O III] $\lambda 5007 / \mathrm{H} \beta$ ratio ( 3.3) than in the main parts of the $\mathrm{H}$ II region $(\sim 2.9)$, which could indicate a slightly higher ionizing level in these regions.

The $\mathrm{H} \alpha / \mathrm{H} \beta$ flux ratio map corrected for differential refraction (Fig. 9) shows a peak ( 3.6) about $3^{\prime \prime}$ South-West of the X-ray position and a second peak ( 3.6) about $10^{\prime \prime}$ West of the Chandra position. The remaining parts of the $\mathrm{H}$ II region have a ratio of $\sim 2.4-2.9$. The second peak is positionally consistent with the peak in the $[\mathrm{O}$ III] $\lambda 5007 / \mathrm{H} \beta$ flux map. Interestingly, the $\mathrm{H}$ II region \#70 seems to be much more extended to the South, as indicated by the red features in the lower panel of the flux ratio map.

To determine the intrinsic flux ratio of the $\mathrm{H}$ II regions we used the case B Balmer recombination decrement $f_{\mathrm{H} \alpha} / f_{\mathrm{H} \beta}=$ 2.85 for $T=10^{4} \mathrm{~K}$ and $N_{\mathrm{e}}=10^{4} \mathrm{~cm}^{-3}$ (Brocklehurst 1971). Assuming that the observed $\mathrm{H} \alpha / \mathrm{H} \beta$ ratio inside the $\mathrm{H}$ II region is due to extinction we obtain $E(B-V)=0.02$ for a ratio of 2.9 and $E(B-V)=0.21$ for a ratio of 3.6. In this case the $\mathrm{H}$ II region would show a larger extinction about $3^{\prime \prime}$ SouthWest of the X-ray position and about $\sim 10^{\prime \prime}$ North-West of the $\mathrm{X}$-ray position. However, the larger $\mathrm{H} \alpha / \mathrm{H} \beta$ ratio in these regions could even be due to higher ionization.

Following Osterbrock (1989) the line flux ratio [S II] $\lambda 6716 /[\mathrm{S} \mathrm{II]} \lambda 6731$ was used to determine the electron density of region \# 70, which is about $200 \mathrm{~cm}^{-3}$. This value is typical for $\mathrm{H}$ II regions. The temperature of the $\mathrm{H}$ II region of $T \sim 15000 \mathrm{~K}$ has been estimated from the line flux ratio [O III] $\lambda 4959+\lambda 5007 /[\mathrm{O}$ III] $\lambda 4363$ (Osterbrock 1989).

The main result of this section is that the emission line flux ratios inside the Holmberg II region \#70, even at the positions of the X-ray and radio sources, are consistent with H II regions.

\subsection{FWHM of emission lines}

To search for dynamical signatures of a black hole we determined the $F W H M$ of the emission lines. The main problem is to correct the observed $F W H M$ for instrumental resolution, which depends on single pixels and the wavelength positions. We have derived the instrumentally corrected FWHM using the $F W H M$ of the night sky lines and the FWHM of the emission lines from calibration lamp exposures. However, we cannot account for all instrumental effects, and these becomes especially significant for the lower resolution MPFS data.

The PMAS FWHM maps and the MPFS FWHM maps (in $\mathrm{km} \mathrm{s}^{-1}$ ) are presented at the bottom of Fig. 4 and in the middle of Figs. 6 and 7, respectively. The color scale of the FWHM images was chosen to show reliable structures of the velocity 
Emission line flux
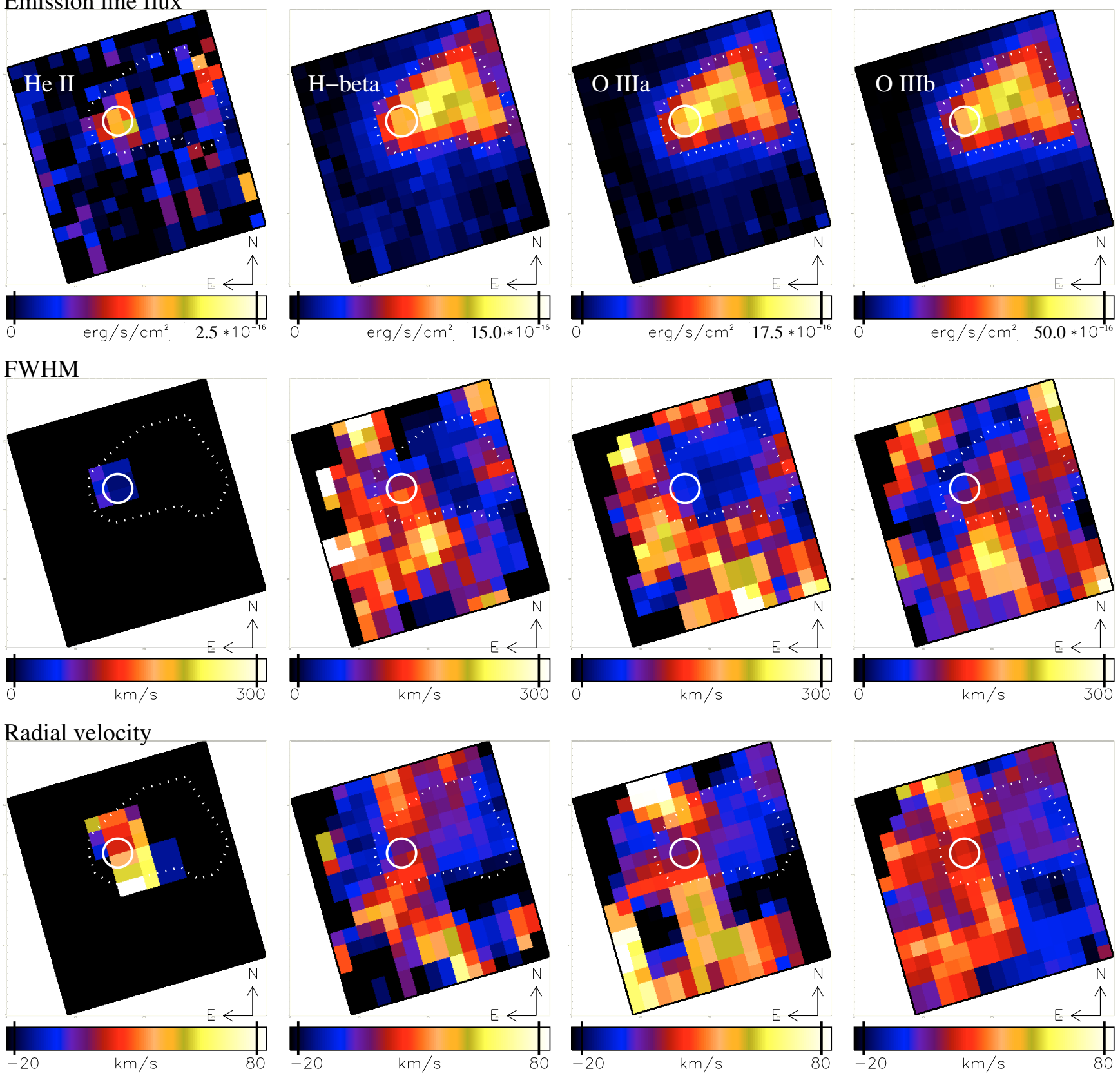

Fig. 6. MPFS emission line flux, FWHM (corrected for instrumental resolution) and radial velocity maps for He II, H $\beta$, [O III] $\lambda 4959$ and [O III] $\lambda 5007$ of the HoII X-1 region. The black color in the flux/radial velocity maps and in the FWHM maps marks regions with no significant line detection and unresolved lines, respectively. The ACIS-S error circle of the X-ray source position is marked. The FOV of the single images is about $15^{\prime \prime} \times 16^{\prime \prime}$. The dotted line in each map marks the area of the H II region given by the [OIII] $\lambda 5007$ line flux larger than $\sim 17 \times 10^{-16} \mathrm{erg} \mathrm{s}^{-1} \mathrm{~cm}^{-2}$. The $F W H M$ and radial velocity images have been smoothed using the mean of a $3 \times 3$ pixel box.

field. Because of the larger angular sampling $\left(0.5^{\prime \prime} /\right.$ pix $)$ and the better spectral resolution the PMAS spectra are better suited to derive the $F W H M$ of the lines. Unfortunately, the PMAS spectra show that the He II $\lambda 4686 /[\mathrm{O}$ III] $\lambda 5007$ emission region, and the PMAS mosaic do not cover the entire Holmberg II region \#70.

The lower panel of Fig. 4 shows that the PMAS FWHM of the $\mathrm{H} \beta$ line inside the $\mathrm{H}$ II region is in general larger than that of the forbidden [O III] $\lambda 5007$ line. The PMAS FWHM of both lines peak around the position of the Chandra ACIS-S error circle.

Because the PMAS and MPFS FWHM maps are given with different scales we present for comparison the peak values of the FWHM inside the H II region derived from both intruments in Table 4. The MPFS FWHM peak values are in agreement with the values derived from PMAS (keeping in mind the lower angular and spectral resolution of the MPFS data), except for [S II] $\lambda 6717$, which is probably blended with the [S II] $\lambda 6731$ emission line. The $F W H M$ of $\mathrm{H} \beta$ derived from 

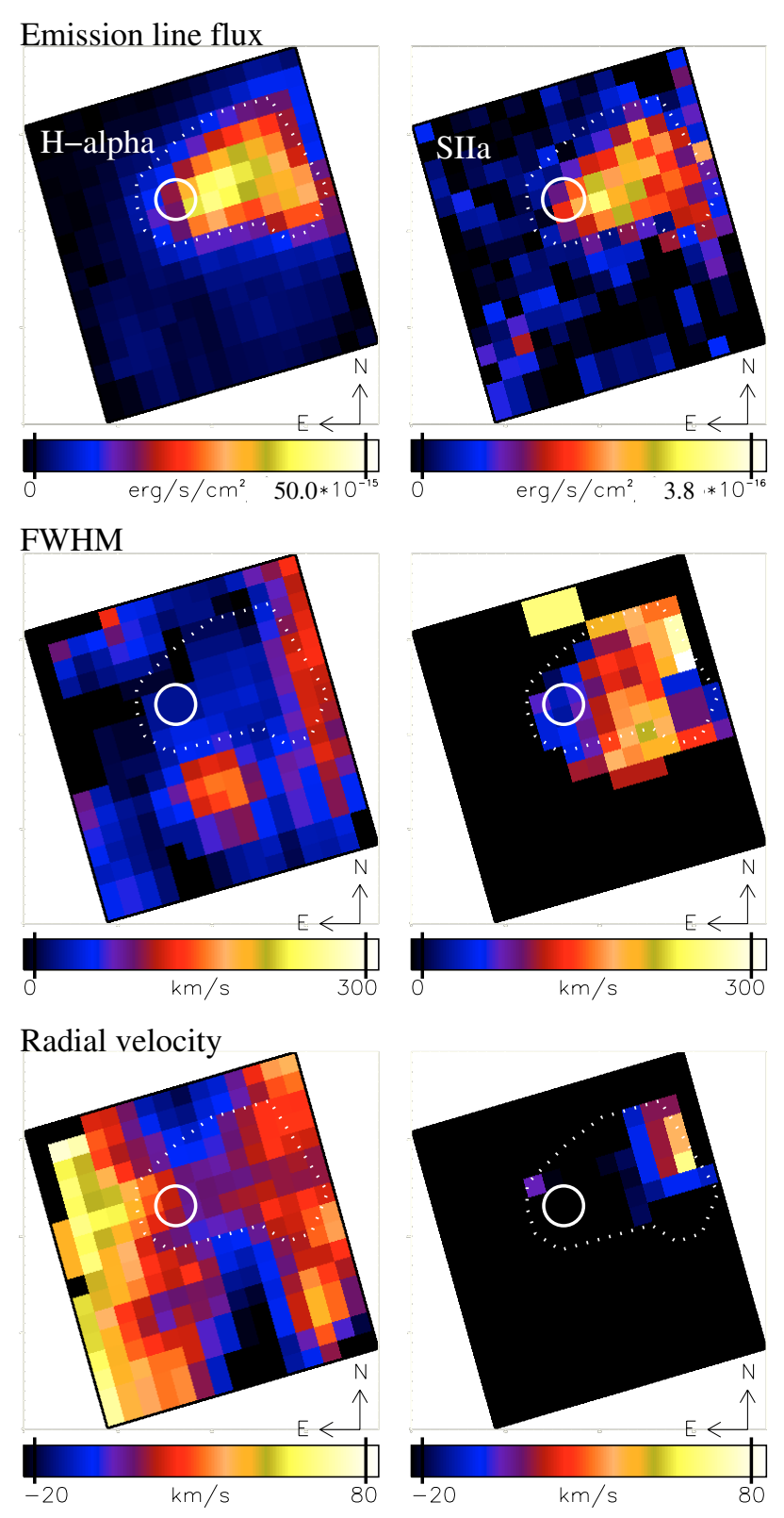

Fig. 7. Same maps as shown in Fig. 6 for $\mathrm{H} \alpha$ and [S II] $\lambda 6717$.

PMAS reaches about $30 \mathrm{~km} \mathrm{~s}^{-1}$ inside the $\mathrm{H}$ II region, but outside the He II sub-region as high as $80 \mathrm{~km} \mathrm{~s}^{-1}$. The $F W H M$ of the $30 \mathrm{~km} \mathrm{~s}^{-1}$ inside the $\mathrm{H}$ II region (which translates into a velocity dispersion of about $13 \mathrm{~km} \mathrm{~s}^{-1}$ ) is consistent with the velocity dispersion measurements of $\sigma_{\mathrm{H} \alpha}=13.0 \pm 0.3 \mathrm{~km} \mathrm{~s}^{-1}$ and $\sigma_{[\mathrm{O} \text { III }] \lambda 5007}=11.3 \pm 0.2 \mathrm{~km} \mathrm{~s}^{-1}$ obtained by Hippelein (1986).

However, the FHWM more than doubles at the X-ray position. From the virial theorem, and assuming that the enclosed mass is related to the velocity change of $\sim 50 \mathrm{~km} \mathrm{~s}^{-1}$ at the distance of $\sim 30 \mathrm{pc}$ the resulting mass of a putative black hole would be about $10^{7} M_{\odot}$. Therefore we believe that the increased $F W H M$ at the location of the He II/X-ray source may be understood as the dynamical influence of the putative black hole similar to the accretion disk wind or jets.

The MPFS FWHM maps show a very complex velocity field South-East of the Chandra position, but outside the main
H II region. Furthermore there is a peak of $\sim 150-200 \mathrm{~km} \mathrm{~s}^{-1}$ about $5^{\prime \prime}$ South-West of the X-ray source position in the MPFS $F W H M$ maps of $\mathrm{H} \beta \lambda 4861$, [O III] $\lambda 5007$, and $\mathrm{H} \alpha$. The complex velocity field outside the $\mathrm{H}$ II region is most probably not related to HoII X-1.

\subsection{Radial velocities}

To determine the radial velocity field around HoII X-1 we used the LSS spectra at the slit positions N2 and N3 and the MPFS data.

In Fig. 11 we show the continuum and background subtracted line isophotes of the 2D-LSS spectra in the He II $\lambda 4686$ emission line. The position of the emission line (or equivalently, the position of the line emitting region) along the wavelength direction (accros the slit) is given as an offset in $\mathrm{km} \mathrm{s}^{-1}$. The position along the slit is given as an offset in arc$\mathrm{sec}$, where the East edge of the "heel" of the compact H II region \#70 is located at an offset of $\sim 17$ " and the He II region at an offset of $\sim 14.5^{\prime \prime}$. The offset values decrease in the NorthWest direction. Both spectra N2 and N3 cover HoII X-1. The He II peak intensity in N2 is 1.4 times greater than that in N3.

The He II region is located near the edge of the H II region. Along the slits we can directly study the structure of the region and we can see the complex structure of the He II emission. Even the shift in the slit position from N2 to N3 $\left(0.6^{\prime \prime}\right)$ results in a noticeable change of the isophote structures.

The line isophote structures across the slits cannot be directly interpreted as radial velocity variations, because the slit width $\left(2^{\prime \prime}\right)$ is larger than the seeing. In the isophotes shown in Fig. 11 the redshift corresponds to a shift of an emission knot covered by the slit in the North-East direction.

There is an additional He II emission region, located about 6-7" ( 100 pc) North-West of the the He II/X-ray source (see Figs. 6 and 10). The same He II emission region is detected in the spectrum N2. The CFHT archival $B$ image in Fig. 2 shows that this is very complex region. The He II emission may originate from Wolf-Rayet stars.

In considering the radial velocity distributions in Fig. 11 we have to remember that the absolute values of the velocities are not correct as they depend on the location of the emission knot within the slit. The complex structure of the radial velocity field is more obvious in the MPFS radial velocity maps in the bottom panel of Figs. 6 and 7. The radial velocity maps of the strong emission lines, $\mathrm{H} \beta$, [O III] $\lambda 4959$ and [O III] $\lambda 5007$ (except of $\mathrm{H} \alpha$ ), show nearly the same structure in the $\mathrm{H}$ II region and its environment. The radial velocity ranges from about -20 to $40 \mathrm{~km} \mathrm{~s}^{-1}$ inside the $\mathrm{H}$ II region, and reaches its maximum around the position of the X-ray source. Crossing the Chandra position nearly in the East-West direction (see the maps of $\mathrm{H} \beta$ to [O III] $\lambda 5007$ in Fig. 6) the radial velocity changes from negative to positive to negative. This behaviour is confirmed by the radial velocities derived from the LSS spectra N2 and N3. On a line crossing the Chandra position in the North-South direction the radial velocity shows only positive values.

As already found for the MPFS spectra, the $\mathrm{H} \beta$ and [OIII] $\lambda 5007$ radial velocities derived from the LSS spectra 


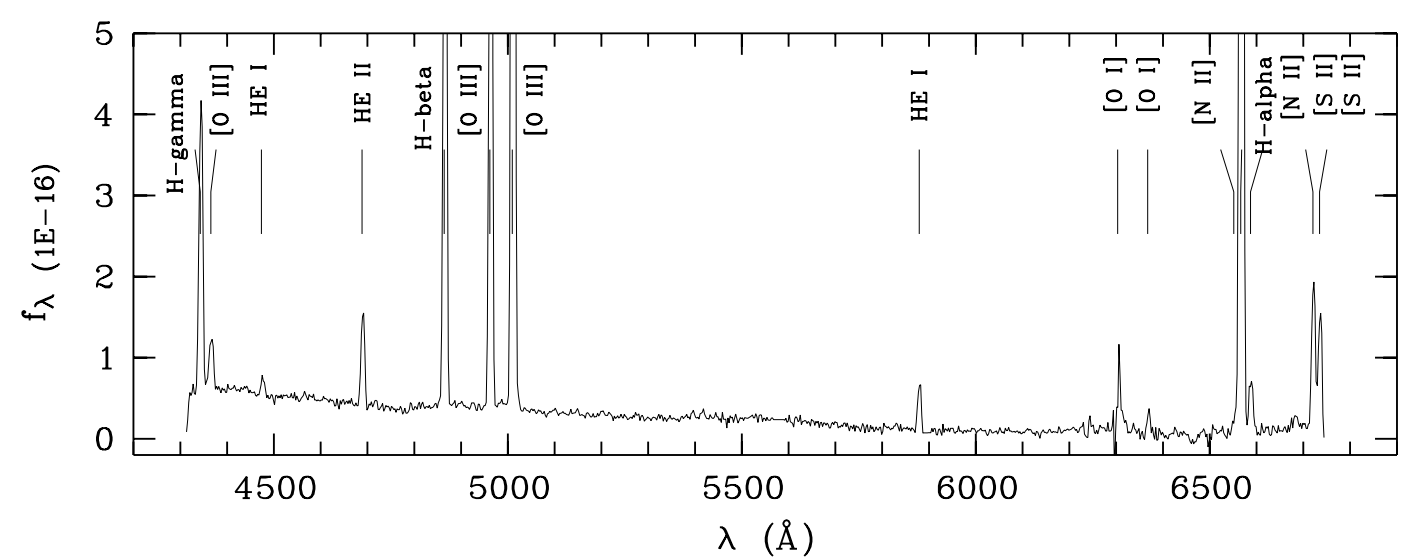

Fig. 8. The average LSS spectrum of the slit positions 2 and 3 (see Fig. 1) at the X-ray source shows, in addition to the lines found in the MPFS spectra, faint [O III] $\lambda 4363$, He I $\lambda 4471$ and [O I] $\lambda 6364$ lines. The continuum emission seems to increase blueward of $\sim 5700 \AA$.
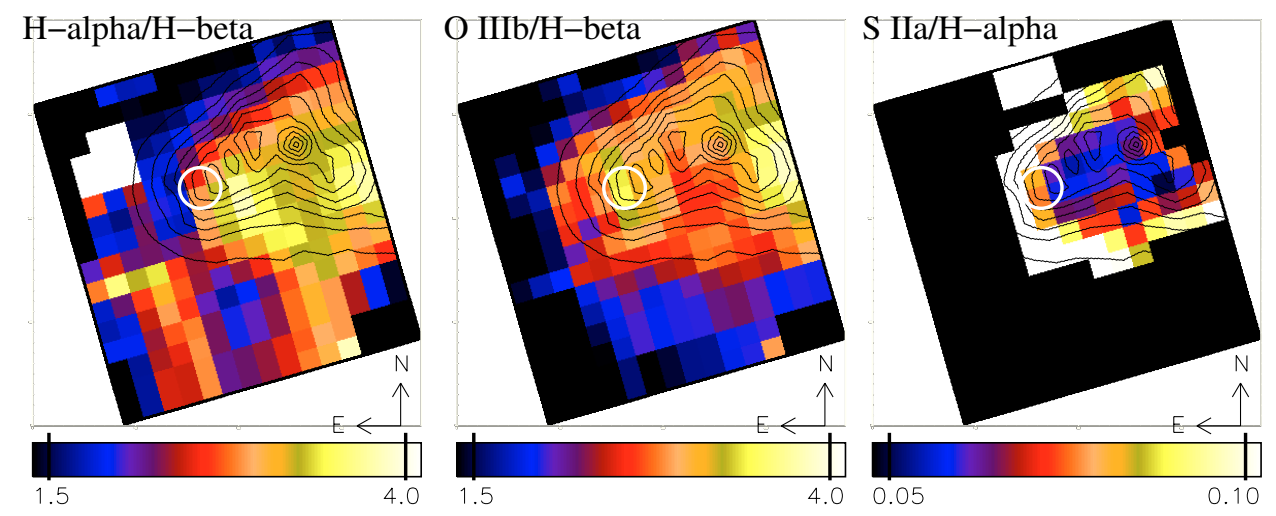

Fig. 9. MPFS line flux ratio maps of Ho II X-1: H $\alpha / \mathrm{H} \beta$, [O III] $\lambda 5007 / \mathrm{H} \beta$ and [S II] $\lambda 6717 / \mathrm{H} \alpha$. The [O III] $\lambda 5007$ line flux contours are overlaid.

Table 2. Relative line fluxes in units of the $\mathrm{H} \beta \lambda 4861$ flux derived from MPFS and LSS data. The fluxes of red forbidden lines in units of the $\mathrm{H} \alpha$ flux are given in brackets.

\begin{tabular}{|c|c|c|}
\hline \multirow[t]{2}{*}{ Line } & \multicolumn{2}{|c|}{ Relative flux } \\
\hline & LSS & MPFS \\
\hline $\mathrm{H} \gamma \lambda 4363$ & 0.45 & - \\
\hline [O III] $\lambda 4363$ & 0.08 & - \\
\hline He I $\lambda 4471$ & 0.08 & - \\
\hline He II $\lambda 4686$ & 0.14 & 0.12 \\
\hline He I $\lambda 4713$ & 0.01 & - \\
\hline He I $\lambda 4922$ & 0.01 & - \\
\hline [O III] $\lambda 4959$ & 1.00 & 1.20 \\
\hline [O III] $\lambda 5007$ & 3.00 & 3.00 \\
\hline He II $\lambda 5412$ & 0.02 & - \\
\hline He I $\lambda 5876$ & 0.07 & - \\
\hline [O I] $\lambda 6300$ & $0.11(0.03)$ & - \\
\hline $\mathrm{H} \alpha \lambda 6563$ & 3.20 & 3.80 \\
\hline$[\mathrm{N} \mathrm{II}] \lambda 6583$ & $0.08(0.02)$ & - \\
\hline He I $\lambda 6678$ & 0.03 & - \\
\hline [S II] $\lambda 6717$ & $0.22(0.07)$ & 0.28 \\
\hline
\end{tabular}

are identical. Because the LSS spectra have higher $\mathrm{S} / \mathrm{N}$ we can study the radial velocity field much better. The LSS spectra show that there is a difference in the He II $\lambda 4686$ and [O III] $\lambda 5007$ radial velocities inside the He II region (see Fig. 12). Moving along the slit N2 from the edge of the "heel" edge (offset position $\sim 16-17^{\prime \prime}$ ) toward the main nebula in the North-West direction, the He II $\lambda 4686$ line shows about the same velocity as the [O III] $\lambda 5007$ line, but it becomes negative $\left(-30 \mathrm{~km} \mathrm{~s}^{-1}\right)$ at an offset position of 12-13". Along the slit N3 the He II behavior is more complex. Its relative radial velocity is about zero at the edge of the "heel", positive (up to $+45 \mathrm{~km} \mathrm{~s}^{-1}$ ) in the middle of the He II region, and negative $\left(-50 \mathrm{~km} \mathrm{~s}^{-1}\right)$ at the offset position of 12-13.5" (North-West of the "heel"). The maximal difference in the radial velocity of the He II $\lambda 4686$ line compared to the [O III] $\lambda 5007$ line is $\pm 50 \mathrm{~km} \mathrm{~s}^{-1}$ on spatial scales of $\pm 2^{\prime \prime}$ ( $\pm 30 \mathrm{pc}$ in projection).

There is a complex radial velocity structure, which may be related to the X-ray source ionizing the surrounding gas. In this case the putative black hole not only ionizes the surrounding gas, but also perturbs the gas dynamically.

\section{Chandra and XMM observations of Holl X-1}

A 5.1 ks Chandra observation of this object was done with the ACIS-S detector. The data were obtained from the public archive and a spectral and spatial analysis was carried out. The log of the Chandra and XMM observations is shown in 
Table 3. Diagnostic emission line ratios from the LSS spectra in logarithmic units.

\begin{tabular}{llc}
\hline \hline No. & ratio & Value \\
\hline 1 & {$[\mathrm{O} \mathrm{III}] \lambda 5007 / \mathrm{H} \beta \lambda 4861$} & 0.48 \\
2 & {$[\mathrm{O} \mathrm{I}] \lambda 6300 / \mathrm{H} \alpha \lambda 6563$} & -1.52 \\
3 & {$[\mathrm{~N} \mathrm{II}] \lambda 6583 / \mathrm{H} \alpha \lambda 6563$} & -1.69 \\
4 & {$[\mathrm{~S} \mathrm{II}] \lambda 6716+\lambda 6731 / \mathrm{H} \alpha \lambda 6563$} & -0.93 \\
5 & {$[\mathrm{~S} \mathrm{II}] \lambda 6716 /[\mathrm{S} \mathrm{II}] \lambda 6731$} & 0.10 \\
6 & {$[\mathrm{O} \mathrm{III}] \lambda 4959+\lambda 5007 /[\mathrm{O} \mathrm{III}] \lambda 4363$} & $\sim 2$ \\
7 & $\mathrm{H} \alpha \lambda 6563 / \mathrm{H} \beta \lambda 4861$ & 0.51 \\
\hline
\end{tabular}

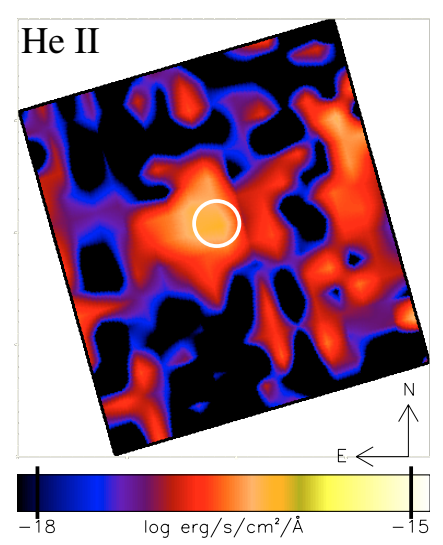

Fig. 10. Monochromatic MPFS images in He II $\lambda 4686$ smoothed with a $2 \times 2$ pixel kernel. The flux is given on logarithmic scale. The Chandra ACIS-S source position is overlaid. The He II image shows a second, probably extended region North-West of the X-ray source, similar to the He II emission line flux map (Fig. 6), and the line intensity plots from the long-slit spectra.

Table 4. Peak values of the FWHM inside the compact H II region \#70 derived from the PMAS and MPFS data.

\begin{tabular}{lcc}
\hline \hline Emission line & \multicolumn{2}{c}{$F W H M_{\text {peak }}$} \\
& PMAS & MPFS \\
\hline He II $\lambda 4686$ & - & $\sim 30 \mathrm{~km} \mathrm{~s}^{-1}$ \\
H $\beta \lambda 4861$ & $\sim 80\left(30^{*}\right) \mathrm{km} \mathrm{s}^{-1}$ & $\sim 80 \mathrm{~km} \mathrm{~s}^{-1}$ \\
{$[$ O III] $\lambda 4959$} & - & $\sim 30 \mathrm{~km} \mathrm{~s}^{-1}$ \\
{$[\mathrm{O}$ III] $\lambda 5007$} & $\sim 30 \mathrm{~km} \mathrm{~s}^{-1}$ & $\sim 80 \mathrm{~km} \mathrm{~s}^{-1}$ \\
$\mathrm{H} \alpha \lambda 6563$ & - & $\sim 30 \mathrm{~km} \mathrm{~s}^{-1}$ \\
{$[\mathrm{~S} \mathrm{II}] \lambda 6716$} & - & $\sim 200 \mathrm{~km} \mathrm{~s}^{-1 * *}$ \\
\hline
\end{tabular}

* Peak value inside the H II but outside the He II region.

** Probably blended with [S II $\lambda 6731]$.

Table 5. Because of the superb spatial resolution of Chandra, we were able to locate HoII X-1 with much better accuracy than in previous observations with ROSAT HRI (MLH01). A two-dimensional Gaussian fit of the Chandra image gives the position of HoII X-1 as RA: $8^{\mathrm{h}} 19^{\mathrm{m}} 29.0^{\mathrm{s}}$, Dec: $+70^{\circ} 42^{\prime} 19^{\prime \prime}$ (J2000). The ROSAT HRI position of RA: $8^{\mathrm{h}} 19^{\mathrm{m}} 29.7^{\mathrm{s}}$ and Dec: $+70^{\circ} 42^{\prime} 18^{\prime \prime}(\mathrm{J} 2000.0)$ agrees well within the uncertainties (Colbert \& Mushotzky 1999).

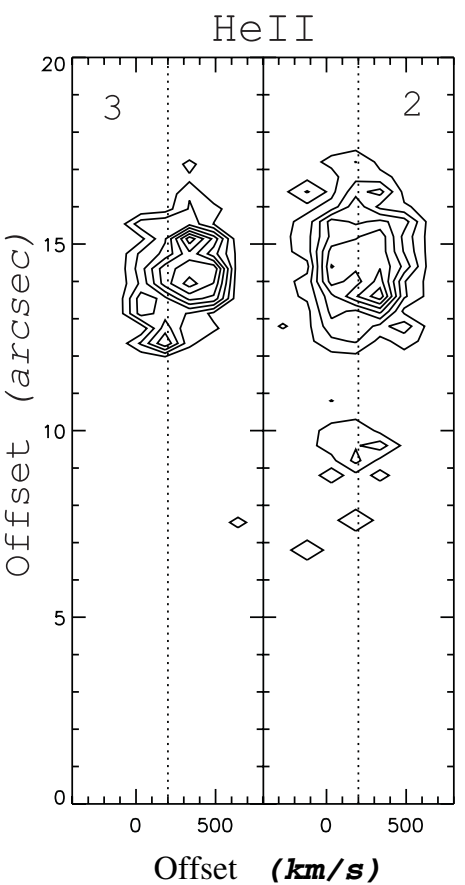

Fig. 11. Isophotes of the 2d-LSS spectra at slit positions N2 and N3 in the He II $\lambda 4686$ emission line. The extended He II region coincident with the X-ray source HoII X-1 is located at an offset value of $\sim 14.5^{\prime \prime}$. A second fainter He II region is detected in the spectrum N2 at an offset value of $\sim 9^{\prime \prime}$ in the North-West direction along the slit.

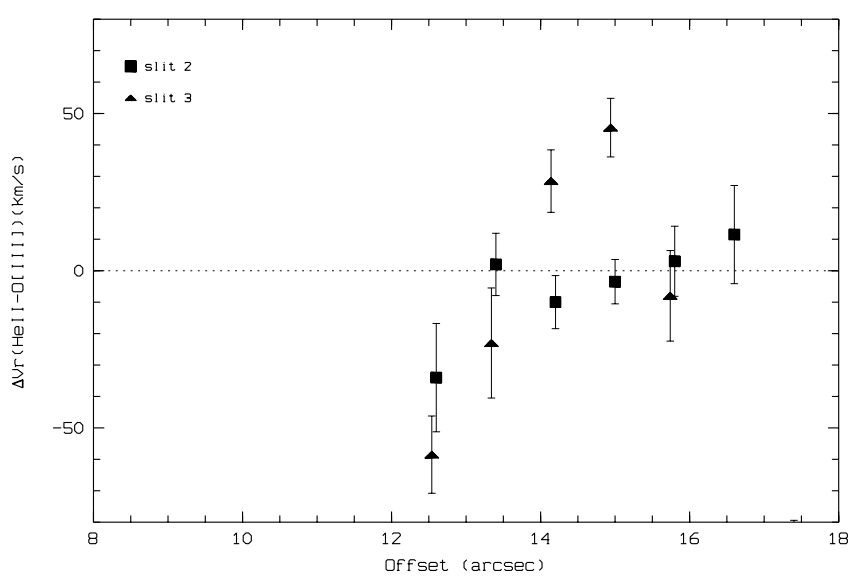

Fig. 12. Radial velocity difference derived from the HeII $\lambda 4686$ and the [O III] $\lambda 5005$ emission lines inside the He II region along the slit position for the N2 and N3. The offset (arcsec) is the same as in Fig. 11, its larger values correspond to the "heel" of the HII region.

Because there are no X-ray sources in the Chandra FOV that can be unambiguously identified with an optical/radio counterpart, the positional accuracy is limited by aspect uncertainties in the Chandra pointing. This is estimated to be $\sim 1^{\prime \prime}$, which we take as the radius of the positional error circle (Fig. 1).

Three XMM observations of HoII X-1 were made with the EPIC PN/MOS detectors (see Table 5). A total of $14.9 \mathrm{ks}$ of good EPIC PN data were obtained after correcting for background flares. A combined events file was derived from the individual events files of the different exposures. The $X M M$ 
Table 5. Log of the analyzed Chandra and XMM Observations.

\begin{tabular}{ccc}
\hline \hline Detector/Modes & Observation & Exposure/Frame \\
\hline Chandra ACIS-S & Date: 02-Nov.-2001 & $5.1 \mathrm{ks}$ \\
Faint/Timed & Seq. 600121 & 1/4 Subarray \\
$X M M$ PN & Date: 10-Apr.-2002 & $5.1 \mathrm{ks}$ \\
Thin & Seq. 0112520601 & PrimeFullWindow \\
$X M M$ PN & Date: 16-Apr.-2002 & $11.1 \mathrm{ks}$ \\
Thin & Seq. 0112522201 & PrimeFullWindow \\
$X M M$ PN & Date: 18-Sep.-2002 & $5.0 \mathrm{ks}$ \\
Thin & Seq. 0112520901 & PrimeFullWindow \\
\hline
\end{tabular}

EPIC PN position of RA: $8^{\mathrm{h}} 19^{\mathrm{m}} 29.1^{\mathrm{s}}$ and Dec: $+70^{\circ} 42^{\prime} 19^{\prime \prime}$ (J2000.0) is in good agreement with the ACIS-S position.

As already mentioned, Tongue \& Westpfahl (1995) have detected a radio peak at 6,20 and $90 \mathrm{~cm}$ wavelengths at the position $8^{\mathrm{h}} 19^{\mathrm{m}} 28^{\mathrm{s}},+70^{\circ} 42^{\prime} 19^{\prime \prime}(\mathrm{J} 2000.0)$. However, because of the low angular resolution of the radio data, it is not clear whether the radio emission $\sim 3^{\prime \prime}$ offset from the X-ray position is coincident with the $\mathrm{X}$-ray source.

\subsection{Spatial analysis}

In Fig. 13 (left panel) we compare the radial profile of HoII X-1 with the ACIS-S Point Spread Function (PSF) at $1 \mathrm{keV}$, where the peak of the source flux lies. The PSF was created using the pre-calculated profiles available in the CIAO package (version 3.0) and normalized to the radial profile of the source at the first annulus outside $3^{\prime \prime}$, because of heavy pile-up in the central region. The $0.5-6 \mathrm{keV}$ source profile was extracted avoiding possible serendipitous sources and the readout streak in the image. The background level was evaluated from an annulus with inner and outer radius of $35^{\prime \prime}$ and $60^{\prime \prime}$, respectively. A hint of extended emission is detected between 4 " -6 " from the source (also emphasized in Fig. 13, right panel), where a possible Westward extinction is evident. The contribution of the extended component to the total flux is estimated to be $\ll 15 \%$. This value is a qualitative estimation of the extended component, since the pile up introduces an error $<20 \%$. A longer exposure would be necessary to put a tighter constraint on the possible extended emission.

\subsection{Spectral analysis}

Here we present a first spectral analysis of the XMM-Newton public data. A more detailed analysis is provided in the following paper by Dewangan et al. (2004).

The EPIC-PN spectrum of HoII X-1 was extracted from a circular region of $\sim 150^{\prime \prime}$ in radius and the background spectrum was obtained from a nearby source free region. For both spectra we use the combined events file from all three observations (see Table 5). Firstly, we fitted a single power law and neutral absorption (fit (A)) to the PN spectrum in the energy range from $0.3-10 \mathrm{keV}$. This gave a fair fit with a $\Gamma \sim 2.8$ power law. The fit parameters are shown in the first entry of Table 6. An obscured single black body, multi-colour disk blackbody
(MCD), and thermal Bremsstrahlung models gave an unacceptable fit $\left(\chi_{\text {red }}^{2}>2\right)$.

The fitted spectrum is steeper than that found in our previous analysis of the ASCA data, which gave $\Gamma \sim 1.9$. In our previous analysis, however, we found a soft excess component in the joint spectral fit using ASCA and ROSAT data (MLH01). The best fit model to the ASCA and ROSAT data was a $k T \sim 0.3 \mathrm{keV}$ thermal plasma, or a $k T_{\text {in }}=0.17 \mathrm{keV}$ disk-blackbody, in addition to a $\Gamma \sim 1.9$ power law. We have applied these models to the EPIC PN spectrum (see Fig. 14) and found that the fit improved significantly. Thus, we consider our previous finding of at least two spectral components in HoII X-1 confirmed. The results of the power law plus thermal fits [fit (B)] are also shown in Table 6. The thermal component is clearly soft (see Fig. 15) and contributes about $18 \%$ to the total $0.3-8.0 \mathrm{keV}$ flux. A power law fit to the soft component is ruled out $\left(\chi^{2}=566\right.$ for 476 d.o.f.).

An absorbed power law and a multicolor disk blackbody (model $\mathrm{C}$ in Table 6) also gives a good fit. This means that the soft component can be as well described by a MCD model. The inner disk temperature is quite low $(k T \sim 0.16 \mathrm{keV})$, which indicates a cool accretion disk. Soft components with cool accretion disks may indicate intermediate-mass black holes with a black hole mass of $M_{\mathrm{BH}} \simeq 10^{3} M_{\odot}$ (Miller et al. 2003).

The excess of cold absorption $\left(N_{\mathrm{H}} \sim 1.6 \times 10^{21} \mathrm{~cm}^{-2}\right)$ over the galactic value $\left(N_{\mathrm{H}} \sim 3.4 \times 10^{20} \mathrm{~cm}^{-2}\right)$ is confirmed by all fits (A to D). The intrinsic cold absorption is in good agreement with recent VLA observations of Holmberg II. Bureau \& Carignan (2002) showed that the compact H II region \#70 is located inside one of the regions with the largest intrinsic hydrogen column density $\left(1.9 \times 10^{21} \mathrm{~cm}^{-2}\right)$.

A power law and a thermal thin plasma model using a solar metal abundance (fit (D)) also gives a resonable fit. The fraction of the $0.3-8.0 \mathrm{keV}$ thermal thin flux (mekal) to the total intrinsic flux is about $9 \%$. The thick plasma thermal emission (bbody) is assumed to originate from the accretion disk around the black hole. Therefore, the thick plasma thermal emission is spatially not resolved. The spatial analysis of the Chandra ACIS-S image suggests a possible extended component with a fraction of less than $15 \%$ of the total $0.3-8.0 \mathrm{keV}$ flux, which would be in agreement with the flux fraction resulting from the thermal thin plasma. This would favor fit (D).

A power law model and a combination of a thick and a thin thermal plasma model (E) gives only a marginal improvement of the fit. The intrinsic flux in the $0.3-8.0 \mathrm{keV}$ band is $9.2 \times 10^{-12} \mathrm{erg} \mathrm{s}^{-1} \mathrm{~cm}^{-2}$ corresponding to a luminosity of $1.1 \times 10^{40} \mathrm{erg} \mathrm{s}^{-1}$. The contribution of the power law component, the thermal black body component, and the thermal thin plasma component to the total $0.3-8.0 \mathrm{keV}$ flux are $77 \%$, $16 \%$ and $7 \%$, respectively. However, a longer EPIC-PN exposure is needed to disentangle a possible extended thermal thin plasma from the point-like power law and thermal thick plasma components.

The spectral analysis of the combined XMM data has confirmed two spectral components in HoII X-1. The hard component is best described by a powerlaw $(\Gamma \sim 2.6)$, and the soft component is fitted by thermal models with relatively low temperatures $(k T \sim 0.14-0.22 \mathrm{keV})$. The intrinsic X-ray 

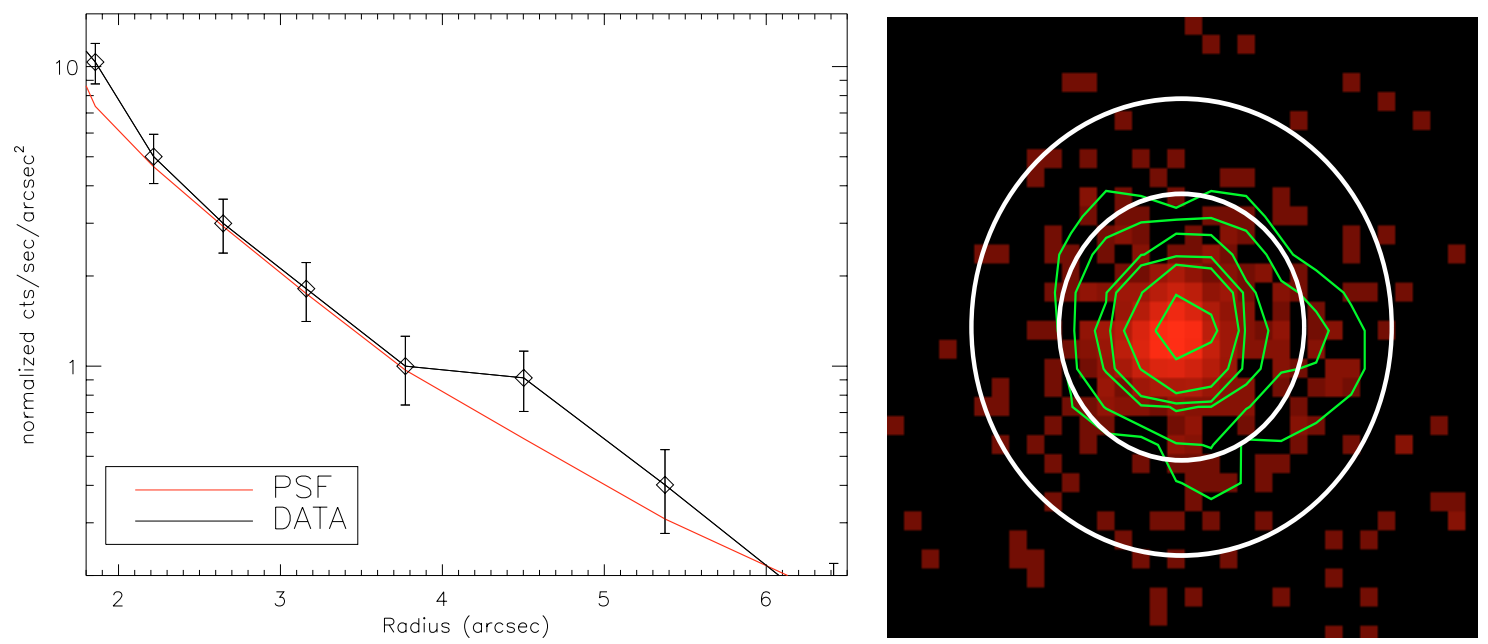

Fig. 13. Left: radial profile of Holmberg II X-1 (diamonds with error bars) compared to the simulated ACIS-S PSF at $1 \mathrm{keV}$. A pixel corresponds to $0.5^{\prime \prime}$. The PSF was normalized to the maximum source counts at the first annulus outside $3^{\prime \prime}$ (6 pixel) in radius. The counts in the radial range between $\sim 4-5^{\prime \prime}$ (5. and 6. radial bins) are marginally above the $1 \mathrm{keV}$ PSF. Right: logarithmically scaled $5 \mathrm{ks}$ Chandra ACIS-S image of HoII X-1 in the $0.3-8.0 \mathrm{keV}$ energy band. The 5, 10, 30, 100, 300, 1000 and 3000 $\sigma$ contours above the logarithmic value of the background are shown (North is up, East is left). The FOV is about $17^{\prime \prime} \times 17^{\prime \prime}$. The circles with 7 and 12 pixel radius mark the annulus with slightly larger counts than expected from to the $1 \mathrm{keV}$ ACIS-S PSF.

luminosity (assuming isotrophic emission) is about $10^{40} \mathrm{erg} \mathrm{s}^{-1}$ in the $0.3-8.0 \mathrm{keV}$ band.

\section{Discussion}

Before we discuss the nature of the ultraluminous X-ray source HoII X-1 we summarize our results:

1. An extended He II $\lambda 4686$ region $(21 \times 47 \mathrm{pc})$ inside an $\mathrm{H}$ II region is positionally coincident with the HoII X-1 source. The existence of such an extended He II emission region is very rare in $\mathrm{H}$ II regions. The He II luminosity agrees well with those published by Pakull \& Mirioni (2002) and Kaaret et al. (2004).

2. Neither the emission line fluxes nor the line flux ratios of the lines other than He II $\lambda 4861$ are correlated with HoII $\mathrm{X}-1$. The emission line flux ratios agree well with those of normal H II regions. This result excludes a SNR nature of the radio source inside the Holmberg II region \#70, which is probably not coincident with the X-ray source.

3. We find a blue extended counterpart for the ULX with a size of $11 \times 14 \mathrm{pc}$, which is most probably a young stellar complex or a cluster. We have derived an X-ray to optical luminosity ratio of $L_{\mathrm{X}} / L_{B} \geq 170$ for the blue stellar complex. The recently published HST ACS images of HoII X-1 (Kaaret et al. 2004) confirm this, however, they suggest a ratio of $L_{\mathrm{X}} / L_{B}=300-400$.

4. The increased $F W H M$ of $\sim 50 \mathrm{~km} \mathrm{~s}^{-1}$ on scale of $\sim 20-30 \mathrm{pc}$ at the position of HoII X-1 is most likely not directly gravitationally related to the rotation around a black hole, as the resulting black hole mass would be $\sim 10^{7} M_{\odot}$. Further, there are radial velocity variations inside the He II region of $\pm 50 \mathrm{~km} \mathrm{~s}^{-1}$ on spatial scales of $\sim 2^{\prime \prime}$ ( $\pm 30 \mathrm{pc}$ in projection $)$. The complex velocity field at the location of the ULX suggests that the putative black hole ionizes the surrounding
HII gas and perturbs it dynamically (via jets or the accretion disk wind).

5. The spatial analysis of the Chandra ACIS-S image gives marginal indications for an extended emission component with an extended flux fraction well below $15 \%$. This could be consistent with the thin thermal plasma component in the $X M M$ spectrum.

6. The XMM EPIC-PN spectrum is well fitted with an absorbed power law $(\Gamma \sim 2.6)$ for the hard component and thermal emission for the soft component, which can be due to a thick plasma (bbody), a thin plasma (mekal), or a multicolor black body disk with relatively low inner disk temperature $\left(k T_{\text {in }} \sim 0.16 \mathrm{keV}\right)$. The intrinsic absorption of $N_{\mathrm{H}} \sim 1.6 \times 10^{21} \mathrm{~cm}^{-2}$ is greater than the Galactic absorption.

There are three models for ULX extensively discussed in the literature:

i) ULX may be black holes of "normal" stellar masses $\left(\sim 10 M_{\odot}\right)$ in binaries, which accrete gas in a supercritical regime. They are SS 433-like objects or microquasars in their transient activity states, whose hard radiation can be collimated (and beamed) along jets and accretion disk axes (Fabrika \& Mescheryakov 2001; King et al. 2001).

ii) ULX may be black holes of a few tens of solar mass $\left(\sim 10 M_{\odot}\right)$; their X-ray emission is from the disk shining at super-Eddington luminosities (Begelman 2002). The slim disk model (Abramowicz et al. 1988; Ebisawa et al. 2003) makes it possible to explain the observed super-Eddington luminosity, hard X-ray spectra and spectral variations.

iii) The ULX may be intermediate mass black holes (IMBHs), $\sim 10^{3} \quad M_{\odot}$ (Colbert \& Mushotzky 1999), which were formed from the very first stars (Madau \& Rees 2001) or in globular clusters (Miller \& Hamilton 2002). The IMBH could accrete gas from a close companion or even from the 
Table 6. Results of the EPIC PN spectral analysis.

\begin{tabular}{|c|c|}
\hline Model & Parameters \\
\hline $\begin{array}{c}\text { PL.Abs. } \\
\text { (A) }\end{array}$ & $\begin{array}{l}\Gamma=2.77_{-0.04}^{+0.04} ; F_{\mathrm{p} 12}=4.38_{-0.13}^{+0.14} \\
N_{\mathrm{H} 20}=16.0_{-0.6}^{+0.7} \\
\chi^{2} / v=\mathbf{1 . 3 0}(621 . / 478)\end{array}$ \\
\hline $\begin{array}{c}\text { PL+Thermal (bbody) } \\
\text { ·Abs. } \\
\text { (B) }\end{array}$ & $\begin{array}{l}\Gamma=2.50_{-0.05}^{+0.09} ; F_{\mathrm{p} 12}=3.38_{-0.18}^{+0.36} \\
k T=.14_{-0.02}^{+0.01} \\
L_{39} / D_{10}^{2}=\left(2.84_{-0.47}^{+1.64}\right) \times 10^{-5} \\
N_{\mathrm{H} 20}=16.4_{-1.1}^{+2.6} \\
\chi^{2} / v=\mathbf{1 . 0 3}(492 . / 476)\end{array}$ \\
\hline $\begin{array}{c}\text { PL+Thermal (diskbb) } \\
\text { ·Abs. } \\
\text { (C) }\end{array}$ & $\begin{array}{l}\Gamma=2.51_{-0.07}^{+0.07} ; F_{\mathrm{p} 12}=3.40_{-0.26}^{+0.29} \\
T \text { in }=.16_{-0.01}^{+0.02} ; \\
\left(R_{\text {in }} / D\right)^{2} \cos \theta=407_{-193}^{+516} \\
N_{\mathrm{H} 20}=18.7_{-0.9}^{+2.3} \\
\chi^{2} / v=\mathbf{1 . 0 4}(496 . / 476)\end{array}$ \\
\hline $\begin{array}{c}\text { PL+Thermal (mekal) } \\
\text { ·Abs. } \\
\text { (D) }\end{array}$ & $\begin{array}{l}\Gamma=2.65_{-.05}^{+.05} ; F_{\mathrm{p} 12}=3.97_{-0.19}^{+0.23} \\
k T=.22_{-0.02}^{+0.02} ; Z=1.0 ; \\
A_{\mathrm{t}}=\left(3.93_{-0.77}^{+1.75}\right) \times 10^{-4} \\
N_{\mathrm{H} 20}=16.2_{-0.9}^{+1.3} \\
\chi^{2} / v=\mathbf{1 . 0 6}(505 . / 476)\end{array}$ \\
\hline $\begin{array}{c}\text { PL+Thermal (mekal+ } \\
\text { bbody) } \cdot \text { Abs. } \\
\text { (E) }\end{array}$ & $\begin{array}{l}\Gamma=2.52_{-.08}^{+.06} ; F_{\mathrm{p} 12}=3.42_{-0.32}^{+.22} ; \\
k T_{\text {mekal }}=.15_{-0.04}^{+0.03} ; Z=1.0 ; \\
A_{\mathrm{t}}=\left(4.6_{-2.0}^{+10.3}\right) \times 10^{-4} ; \\
k T_{\text {bbody }}=.15_{-0.03}^{+0.01} ; \\
L_{39} / D_{10}^{2}=\left(2.2_{-0.6}^{+1.3}\right) \times 10^{-5} ; \\
N_{\mathrm{H} 20}=16.9_{-1.7}^{+1.3} ; \\
\chi^{2} / v=\mathbf{0 . 9 8}(463 . / 474)\end{array}$ \\
\hline
\end{tabular}

Fit parameters are shown with $90 \%$ errors $\left(\Delta \chi^{2}=2.7\right)$. The model and parameter definitions are: model components - PL: power-law with a photon index of $\Gamma$ and a $0.5-2 \mathrm{keV}$ flux of $F_{\mathrm{p} 12}$ in units of $10^{-12} \mathrm{erg} \mathrm{cm}^{-2} \mathrm{~s}^{-1}$. Thermal - thin plasma: thermal plasma using the XSPEC mekal model with a plasma temperature $k T[\mathrm{keV}]$, a metal abundance $Z=1$ in solar units, and the normalization defined by XSPEC $A_{\text {th }}$. Thermal - multi color disk: the multicolor disk (Mitsuda et al. 1984) distributed as the XSPEC model (diskbb) with an inner disk temperature $k T_{\text {in }}$ and with an normalization defined as $\left(R_{\text {in }} / D\right) \cos \theta$, where $R_{\text {in }}$ is the inner disk radius and $D$ is the distance to the source in $10 \mathrm{kpc}$ units, and $\theta$ is the viewing angle of the disk axis. Thermal - thick plasma: thermal thick plasma using the XSPEC bbody model with a plasma temperature $k T[\mathrm{keV}]$, with a normalization defined as $L_{39} / D_{10}^{2}$, where $L_{39}$ is the source luminosity in units of $10^{39} \mathrm{erg} \mathrm{s}^{-1}$ and $D_{10}$ is the distance to the source in units of $10 \mathrm{kpc}$. Abs: absorption by neutral gas using the XSPEC model wabs (Morrison R. McCammon D. 1983, ApJ 270 190) with hydrogen column density $N_{\mathrm{H} 20}\left[10^{20} \mathrm{~cm}^{-2}\right]$.

interstellar medium and become bright X-ray sources when they are in dense gas environments. Spectra of some ULX (Miller et al. 2003) support the idea that they are IMBHs.

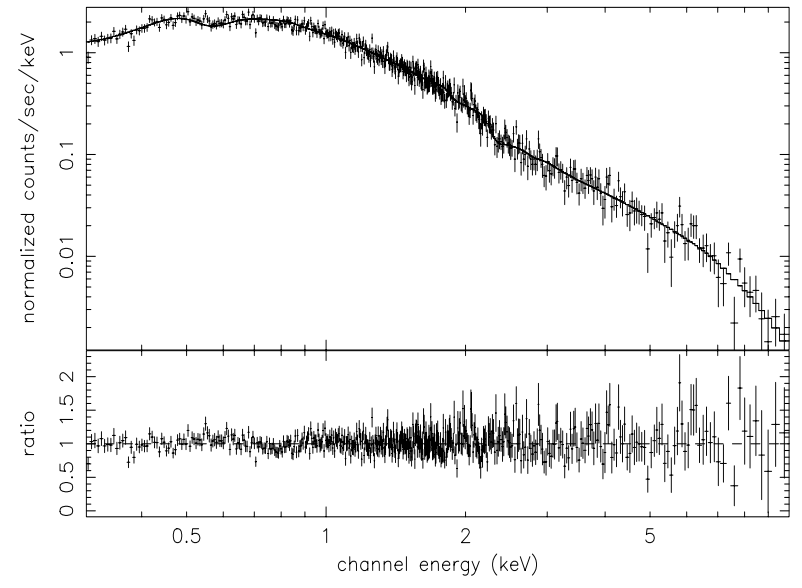

Fig. 14. The X-ray pulse-height spectrum of XMM EPIC-PN is shown with folded models for fit (B). The lower panel shows the data/model ratios. Pulse height spectra are re-binned for display and $1 \sigma$ error bars are attached to data points.

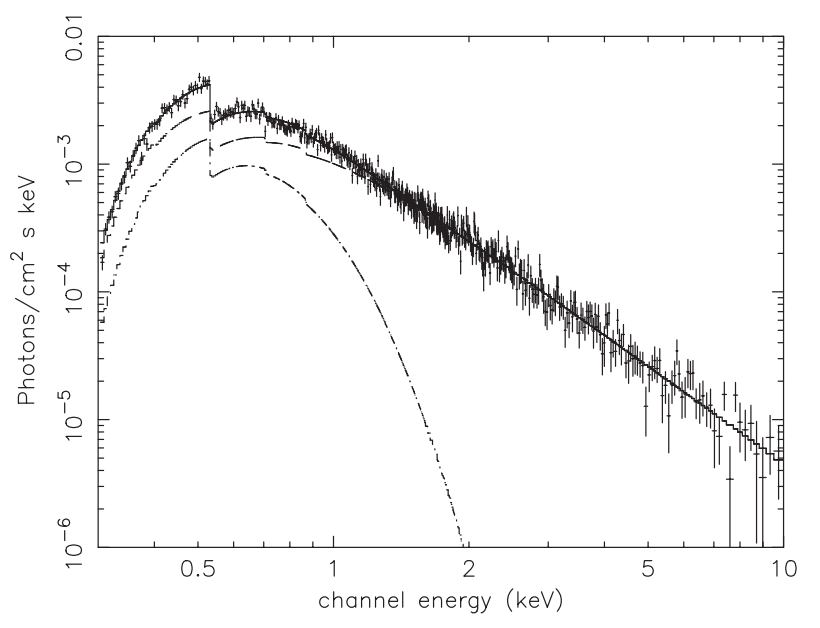

Fig. 15. Unfolded $X M M$ EPIC-PN spectrum showing the best fit model (B). The thermal black body component contributes about $18 \%$ to the total $0.3-8.0 \mathrm{keV}$ flux, and is clearly soft $(<2 \mathrm{keV})$.

Substantial extended He II $\lambda 4886$ emission has been confirmed inside the compact H II region \#70 at the position of HoII X-1. Such strong He II $\lambda 4886$ emission is produced by reprocessing of the X-ray source located inside the nebula. Pakull \& Mirioni (2002) have concluded that the optical radiation is consistent with a quasi-isotrophic X-ray source and no significant beaming is required. In this case the source is really bright in X-rays $\left(L_{\mathrm{X}} \sim 10^{40} \mathrm{erg} / \mathrm{s}\right)$, which supports an IMBH interpretation (iii). The extended blue counterpart of HoII X-1 suggests that the IMBH is located in a young stellar complex or cluster.

The IMBH may disturb the velocity field (via the accretion disk wind or jets) of the $\mathrm{H}$ II region at its position, where we have found an increase of the velocity dispersion, and a radial velocity variation accross the He II $\lambda 4686$ region. The amplitude of the radial velocity gradient accross the He II $\lambda 4686$ region is $\pm 50 \mathrm{~km} \mathrm{~s}^{-1}$ on spatial scales of $\pm 2^{\prime \prime}$. This means that the ULX has not only ionized the surrounding gas, but also interacted with it dynamically. 
The X-ray properties of HoII X-1 seem to agree with the IMBH interpretation (iii). The soft component of the X-ray spectrum of HoII X-1 can be described by a MCD model with a inner disk temperature of $k T \sim 0.16 \mathrm{keV}$. Such a cool accretion disk is an indication of an intermediate-mass black hole with a black hole mass of $M_{\mathrm{BH}} \simeq 10^{3} M_{\odot}$ (Miller et al. 2003). A further indication of an IMBH is the long-term variability of HoII X-1 (see MLH01 and Dewangan et al. 2004).

The optical/X-ray properties of HoII X-1 seem to favour the IMBH nature of the object. High angular resolution integral field observation with HST or ground based near-infrared integral field spectroscopy using adaptive optics at an 8-m class telescope is needed to prove the IMBH nature of HoII X-1, and would make it possible to put tighter constraints on the velocity structure of the gas and the stars inside the cluster around HoII $\mathrm{X}-1$, and could enable us to measure the mass of the IMBH.

Acknowledgements. T. Becker and M. M. Roth were Visiting Astronomers, German-Spanish Astronomical Centre, Calar Alto, operated by the Max-Planck-Institute for Astronomy, Heidelberg, jointly with the Spanish National Commission for Astronomy.

The authors thank the Canadian Astronomy Data Center, which is operated by the Dominion Astrophysical Observatory for the National Resarch Council of Canada's Herzberg Institute of Astrophysics. This research has made use of the USNOFS Image and Catalogue Archive operated by the United States Naval Observatory, Flagstaff Station (http://www.nofs .navy.mil/data/fchpix/). The work has been supported by Russian RFBR grants N03-02-16341, 04-02-16349, and INTAS grant YSF 2002-281.

We thank L. Gallo for his valuable comments and suggestions. Further we want to thank V. Goranskii for help with the photometry of the optical counterpart.

\section{References}

Abramowicz, M. A., Czerny, B., Lasota, J. P., \& Szuszkiewicz, E. 1988, ApJ, 332, 646A

Afanasiev, V. L., Vlasyuk, V. V., \& Green, R. F. 1995a, Tridimensional Optical Spectroscopic Methods in Astrophysics, ASP Conf. Ser., 71, Proc. IAU Coll., 149, held in Marseille, France, March 22-25, 1994, ed. G. Comte, \& M. Marcelin (San Francisco, California: ASP), 266

Afanasiev, V. L., Burenkov, A. N., Vlasuk, V. V., \& Drabek, S. V. 1995b, SAO RAS internal report, No. 243

Allen, C. W. 1973, Astrophysical Quantities (The Athlone Press)

Angelini, L., Loewenstein, M., \& Mushotzky, R. F. 2001, ApJ, 557, L35

Becker, T. 2002, Ph.D. Thesis, Univ. Potsdam

Begelman, M. C. 2002, ApJ, 567, L97

Brocklehurst, M. 1971, MNRAS, 153, 471

Bureau, M., \& Carignan, C. 2002, AJ, 123, 1316
Colbert, E. J. M., \& Mushotzky, R. F. 1999, ApJ, 519, 89

Dewangan, G. C., Miyaji, T., Griffiths, R. E., \& Lehmann, I. 2004, ApJ, 608, 57

Dopita, M. A., \& Sutherland, R. S. 1995, ApJ, 446, 75

Ebisawa, K., Źycki, P., Kubota, A., Mizuno, T., \& Watari, K. 2003, ApJ, 597, 780

Fabbiano, G. 1989, ARA\&A, 27, 87

Fabrika, S., \& Mescheryakov, A. 2001, Galaxies and their Constituents at the Highest Angular Resolutions, Proc. IAU Symp., 205, ed. R. T. Schilizzi, 268

Filippenko, A. V. 1982, PASP, 94, 715

Foschini, L., Di Cocco, G., \& Ho, L. C. 2002, A\&A, 392, 817

Hippelein, H. H. 1986, A\&A, 160, 374

Hodge, P., Strobel, N. V., \& Kennicutt, R. C. 1994, PASP, 106, 309

Jedrezejewski, R. I. 1987, MNRAS, 311, 576

Kaaret, P., Ward, M. J., \& Zezas, A. 2004, MNRAS, 351, 83

Karachentsev, I. D., Dolphin, A. E., Geisler, D., et al. 2002, A\&A, 383,125

King, A. R., Davies, M. B., Ward, M. J., Fabbiano, G., \& Elvis, M. 2001, ApJ, 552, 109

Madau, P., \& Rees, M. J. 2001, ApJ, 551, L27

Miller, B. W. 1995, ApJ, 446, 75

Miller, M. C. 2002, ApJ, 330, 232

Miller, J. M., Fabbiano, G., Miller, M. C., \& Fabian, A. C. 2003, AJ, 585, L37

Miller, M. C., \& Colbert, E. J. M. 2004, Int. J. Mod. Phys. D, 13, 1

Mitsuda, K., Inoue, H., \& Koyama, K. 1984, PASJ, 36, 741

Miyaji, T., Lehmann, I., \& Hasinger, G. 2001, AJ, 121, 3041

Odewahn, S. C., Bryja, C., \& Humphreys, R. M. 1992, PASP, 104, 553

Osterbrock, D. E. 1989, Astrophysics of Gaseous Nebulae and Active Galactic Nuclei (Mill Valley, California: University Science Books)

Smith, R. C., Kirshner, R. P., Blair, W. P., Long, K. S., \& Winkler, P. F. 1993, ApJ, 407, 564

Pakull, M. W., \& Mirioni, L. 2002, Proc. Symp., New Visions of the X-ray Universe in the XMM-Newton and Chandra Era, 2630 November 2001 [arXiv: astro-ph/0202488]

Press, H. W., Teukolski, S. A., Vetterling, W. T., \& Flannery, B. P. 1992, Numerical Recipes in FORTRAN, example book, sec. edition (Cambridge Univ. Press)

Roth, M. M., Bauer, S. M., Dionies, F., et al. 2000, in Proc. Optical and IR Telescope Instrumentation and Detectors, ed. M. Iye, \& A. F. M. Moorwood, SPIE008, 277

Sánchez, S. F., \& González-Serrano, J. I. 2003, A\&A, 406, 435

Schlegel, D. J., Finkbeiner, D. P., \& Davis, M. 1998, ApJ, 500, 525

Tongue, T. D., \& Westpfahl, D. J. 1995, AJ, 109, 2462

Veilleux, S., \& Osterbrock, D. E. 1987, ApJS, 63, 295

Wang, Q. D. 2002, MNRAS, 332, 764

Wu, H., Xue, S. J., Xia, X. Y., Deng, Z. G., \& Mao, S. 2002, ApJ, 576, 738

Zesas, A. L., Geogantopoulos, I., \& Ward, M. J.1999, MNRAS, 308, 302 